hep-th/9408083

TIFR-TH-94-19

\title{
STRONG-WEAK COUPLING DUALITY IN THREE DIMENSIONAL STRING THEORY
}

\author{
ASHOKE SEN ${ }^{\star}$ \\ Tata Institute of Fundamental Research, Homi \\ Bhabha Road, Bombay 400005, India
}

\begin{abstract}
Following an old result of Marcus and Schwarz we argue that in the heterotic string theory compactified on a seven dimensional torus, the target space duality group $\mathrm{O}(7,23 ; \mathrm{Z})$ and the strong-weak coupling duality transformations combine into the group $\mathrm{O}(8,24 ; \mathrm{Z})$. We discuss symmetry of the combined spectrum of elementary particles and solitons in the theory, and also show that the existence of this symmetry predicts the number of harmonic forms on the moduli space of periodic arrays of BPS monopoles in $(3+1)$ dimensions. Finally, we show that the $\mathrm{O}(8,24 ; \mathrm{Z})$ transformations relate the soliton solutions of Dabholkar et. al. representing the fundamental string to the soliton solutions of Greene et. al. representing 'stringy cosmic strings'.
\end{abstract}

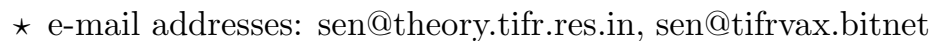




\section{Introduction and Summary}

There is mounting evidence $[1-10]$ that the heterotic string theory compactified on a six dimensional torus is invariant under an $\mathrm{SL}(2, \mathrm{Z})$ group of transformations that acts non-trivially on the coupling constant of the theory. Some of the consequences of this symmetry has been analyzed in refs.[11][12]. It is natural to ask if this symmetry is present for more realistic compactification of the theory, say for compactification on a Calabi-Yau manifold. At present there does not seem to be a way to answer this question, since testing this symmetry requires computing some observables (e.g. mass spectrum, partition function etc.) exactly, i.e. without using perturbation theory. There are certain non-renormalization theorems $[13-15]$ in the toroidal compactification of the heterotic string theory that allows us to calculate some quantities in the theory exactly, and hence test the symmetry. Also, in the field theory limit, the partition function of a twisted version of the theory is exactly calculable [9]. No such powerful non-renormalization theorem exists in the $N=1$ supersymmetric compactification of the heterotic string theory. $N=2$ supersymmetric compactifications provide an intermediate case, and the recent results of Seiberg and Witten [16] might be useful in analyzing the structure of duality transformations in heterotic string theory compactified on $K 3 \times S^{1} \times S^{1}$ [17]. (For a recent analysis of this problem see ref.[18].)

In the absence of a mechanism to test this symmetry in a realistic compactification scheme, we can at least try to ask if this symmetry is present in other (non-realistic) compactification of the theory. This might give a clue to how this symmetry might depend on the compactification scheme. With this in mind, we shall study the duality group of heterotic string theory compactified on a seven dimensional torus. In the low energy limit, this will result in a three dimensional supergravity theory with eight local supersymmetries.

Such theories were analyzed long ago by Marcus and Schwarz [19]. The only massless bosonic fields in this theory are the spin two (non-propagating) graviton, and a set of scalar fields; this is due to the fact that in three dimensions vector 
fields are dual to the scalar fields and hence all vector fields can be traded in for scalar fields. It was shown in Ref.[19] that the scalar fields parametrize the coset $\mathrm{O}(8,24) / \mathrm{O}(8) \times \mathrm{O}(24)$, and that the effective action is invariant under $\mathrm{O}(8,24)$ transformation (see also [20]). The duality group of the full string theory is expected to be a subgroup of this $\mathrm{O}(8,24)$ group. Of this, a subgroup $\mathrm{O}(7,23 ; \mathrm{Z})$ is already known to be a symmetry of the theory, - this is the standard target space duality group for the compactification of the heterotic string on a seven dimensional torus [21]. In this paper we shall argue that the duality group in fact is much larger, - $\mathrm{O}(8,24 ; \mathrm{Z})$, - with a generic element of $\mathrm{O}(8,24 ; \mathrm{Z})$ acting non-trivially on the coupling constant of the theory. ${ }^{\dagger}$

An intuitive understanding of this large group of symmetries can be given as follows. The three dimensional theory can be regarded as a four dimensional theory compactified on a circle. Since the strong-weak coupling duality group SL(2,Z) does not act on space-time, we would expect that this duality symmetry will remain unbroken even when we compactify one of the three spatial directions. There are, however, seven independent ways of regarding the three dimensional theory as a four dimensional theory compactified on a circle, since any of the seven compact directions could be regarded as the non-compact direction in the original four dimensional theory before compactification. Thus the three dimensional theory, besides possessing the usual $\mathrm{O}(7,23 ; \mathrm{Z})$ target space duality symmetry, is expected to be invariant under seven different $\mathrm{SL}(2, \mathrm{Z})$ symmetry groups. The transformations belonging to these different groups do not commute with each other, and are thus expected to generate a big non-abelian discrete group. As we shall see, the $\mathrm{O}(8,24 ; \mathrm{Z})$ group contains the $\mathrm{O}(7,23 ; \mathrm{Z})$ group, as well as each of the $\mathrm{SL}(2, \mathrm{Z})$ groups, as its subgroup.

If there is a general lesson to be learned from the analysis of this paper, it is probably that for a generic string compactification we should not expect the duality group to be a product of the target space duality group and the coupling

\footnotetext{
$\dagger$ This was already hinted in ref.[5].

$\ddagger$ This argument has been developed independently by E. Witten (private communication).
} 
constant duality group. In particular, for string theory compactified on a CalabiYau manifold [22], or for more general $(0,2)$ string compactification [23], the duality transformations may mix the axion-dilaton field with the other moduli fields in a non-trivial manner. Other interesting compactifications of the theory to three dimensions [24] may also provide a good laboratory for testing this phenomenon.

The paper is organized as follows. In sect. 2 we carry out the dimensional reduction of the ten dimensional theory to three dimensions, and show that the resulting theory is manifestly $\mathrm{O}(8,24)$ invariant. We also identify an $\mathrm{O}(8,24 ; \mathrm{Z})$ subgroup of this which we claim to be an exact symmetry of the full string theory. In sect.3 we show that various classical solutions in the three dimensional theory, representing periodic arrays of monopoles in the four dimensional theory, are related to elementary string states via specific $\mathrm{O}(8,24 ; \mathrm{Z})$ transformations. We discuss the quantization of some of these classical solutions in sect.4 and show that the $\mathrm{O}(8,24 ; \mathrm{Z})$ symmetry of the three dimensional theory makes definite prediction about the existence of harmonic forms in the moduli space of these solutions. In sect.5 we show that two of the hitherto unrelated, but similar solutions in string theory, - the 'stringy cosmic string' solution of ref.[25], and the 'fundamental string' solution of ref.[15], - are in fact related by a $Z_{2}$ subgroup of the $\mathrm{O}(8,24 ; \mathrm{Z})$ group. In this section we also suggest a way by which $\mathrm{O}(8,24 ; \mathrm{Z})$ invariance of the theory might resolve the usual infra-red problems associated with charged particles in three dimensions. In the three appendices we discuss various properties of periodic arrays of BPS monopole solutions in $(3+1)$ dimensions, since these solutions provide most of the testing ground for the $\mathrm{O}(8,24 ; \mathrm{Z})$ symmetry of the three dimensional theory. 


\section{2. $\mathrm{O}(8,24)$ Invariant Effective Action}

We shall begin by discussing how the dynamics of massless fields in the heterotic string theory, compactified on a seven dimensional torus, is described by an $\mathrm{O}(8,24)$ invariant effective action. This will also establish the relationship between the fields that transform naturally under $\mathrm{O}(8,24)$, and the fields appearing from the dimensional reduction of the $N=1$ supergravity theory in ten dimensions, and will be useful to us later for studying the $\mathrm{O}(8,24 ; \mathrm{Z})$ transformation properties of elementary string excitations and solitons.

We start with the $N=1$ supergravity theory coupled to $N=1$ super YangMills theory in ten dimensions, and dimensionally reduce the theory from ten to three dimensions. Since at a generic point in the moduli space only the abelian gauge fields give rise to massless fields in three dimensions, it is enough to restrict to the $\mathrm{U}(1)^{16}$ part of the ten dimensional gauge group. The ten dimensional action is given by,

$$
\begin{array}{r}
C \int d^{10} z \sqrt{-G^{(10)}} e^{-\Phi^{(10)}}\left(R^{(10)}+G^{(10) M N} \partial_{M} \Phi^{(10)} \partial_{N} \Phi^{(10)}\right. \\
\left.-\frac{1}{12} H_{M N P}^{(10)} H^{(10) M N P}-\frac{1}{4} F_{M N}^{(10) I} F^{(10) I M N}\right)
\end{array}
$$

where $G_{M N}^{(10)}, B_{M N}^{(10)}, A_{M}^{(10) I}$, and $\Phi^{(10)}$ are ten dimensional metric, anti-symmetric tensor field, $\mathrm{U}(1)$ gauge fields and the scalar dilaton field respectively $(0 \leq M, N \leq$ $9,1 \leq I \leq 16)$, and,

$$
\begin{aligned}
F_{M N}^{(10) I} & =\partial_{M} A_{N}^{(10) I}-\partial_{N} A_{M}^{(10) I} \\
H_{M N P}^{(10)} & =\left(\partial_{M} B_{N P}^{(10)}-\frac{1}{2} A_{M}^{(10) I} F_{N P}^{(10) I}\right)+\text { cyclic permutations of } M, N, P .
\end{aligned}
$$

We have ignored the fermion fields in writing down the action (2.1). $C$ is an arbitrary constant that we shall choose appropriately for later convenience. This constant can always be absorbed into a shift in the dilaton field. 
The dimensional reduction of this theory can be carried out following the procedure of refs.[26][27]. We shall follow the notation of ref.[6] and introduce the 'three dimensional fields' $\widehat{G}_{m n}, \widehat{B}_{m n}, \widehat{A}_{m}^{I}, \Phi, A_{\mu}^{(a)}, g_{\mu \nu}$ and $B_{\mu \nu}(1 \leq m \leq 7$, $0 \leq \mu \leq 2,1 \leq a \leq 30)$ through the relations

$$
\begin{aligned}
& \widehat{G}_{m n}=G_{m+2, n+2}^{(10)}, \quad \widehat{B}_{m n}=B_{m+2, n+2}^{(10)}, \quad \widehat{A}_{m}^{I}=A_{m+2}^{(10) I}, \\
& A_{\mu}^{(m)}=\frac{1}{2} \widehat{G}^{m n} G_{n+2, \mu}^{(10)}, \quad A_{\mu}^{(I+14)}=-\left(\frac{1}{2} A_{\mu}^{(10) I}-\widehat{A}_{n}^{I} A_{\mu}^{(n)}\right), \\
& A_{\mu}^{(m+7)}=\frac{1}{2} B_{(m+2) \mu}^{(10)}-\widehat{B}_{m n} A_{\mu}^{(n)}+\frac{1}{2} \widehat{A}_{m}^{I} A_{\mu}^{(I+14)}, \\
& G_{\mu \nu}=G_{\mu \nu}^{(10)}-G_{(m+2) \mu}^{(10)} G_{(n+2) \nu}^{(10)} \widehat{G}^{m n}, \\
& B_{\mu \nu}=B_{\mu \nu}^{(10)}-4 \widehat{B}_{m n} A_{\mu}^{(m)} A_{\nu}^{(n)}-2\left(A_{\mu}^{(m)} A_{\nu}^{(m+7)}-A_{\nu}^{(m)} A_{\mu}^{(m+7)}\right), \\
& \Phi=\Phi^{(10)}-\frac{1}{2} \ln \operatorname{det} \widehat{G}, \quad g_{\mu \nu}=e^{-2 \Phi} G_{\mu \nu}, \\
& \quad 1 \leq m, n \leq 7, \quad 0 \leq \mu, \nu \leq 2, \quad 1 \leq I \leq 16 .
\end{aligned}
$$

Here $\widehat{G}^{m n}$ denotes the inverse of the matrix $\widehat{G}_{m n}$. We now combine the scalar fields $\widehat{G}_{m n}, \widehat{B}_{m n}$, and $\widehat{A}_{m}^{I}$ into an $O(7,23)$ matrix valued scalar field $M$. For this we regard $\widehat{G}_{m n}, \widehat{B}_{m n}$ and $\widehat{A}_{m}^{I}$ as $7 \times 7,7 \times 7$, and $7 \times 16$ matrices respectively, and $\widehat{C}_{m n}=\frac{1}{2} \widehat{A}_{m}^{I} \widehat{A}_{n}^{I}$ as a $7 \times 7$ matrix, and define $M$ to be the $30 \times 30$ dimensional matrix

$$
M=\left(\begin{array}{ccc}
\widehat{G}^{-1} & \widehat{G}^{-1}(\widehat{B}+\widehat{C}) & \widehat{G}^{-1} \widehat{A} \\
(-\widehat{B}+\widehat{C}) \widehat{G}^{-1} & (\widehat{G}-\widehat{B}+\widehat{C}) \widehat{G}^{-1}(\widehat{G}+\widehat{B}+\widehat{C}) & (\widehat{G}-\widehat{B}+\widehat{C}) \widehat{G}^{-1} \widehat{A} \\
\widehat{A}^{T} \widehat{G}^{-1} & \widehat{A}^{T} \widehat{G}^{-1}(\widehat{G}+\widehat{B}+\widehat{C}) & I_{16}+\widehat{A}^{T} \widehat{G}^{-1} \widehat{A}
\end{array}\right)
$$

satisfying

$$
M L M^{T}=L, \quad M^{T}=M, \quad L=\left(\begin{array}{ccc}
0 & I_{7} & 0 \\
I_{7} & 0 & 0 \\
0 & 0 & -I_{16}
\end{array}\right)
$$

where $I_{n}$ denotes the $n \times n$ identity matrix. 
The effective action that governs the dynamics of the massless fields in the three dimensional theory is obtained by substituting in Eq.(2.1) the expressions for the ten dimensional fields in terms of the three dimensional fields, and taking all field configurations to be independent of the internal coordinates. The result is

$$
\begin{aligned}
S=\frac{1}{4} \int d^{3} x \sqrt{-g}\left[R_{g}-g^{\mu \nu} \partial_{\mu} \Phi \partial_{\nu} \Phi-\frac{1}{12} e^{-4 \Phi} g^{\mu \mu^{\prime}} g^{\nu \nu^{\prime}} g^{\rho \rho^{\prime}} H_{\mu \nu \rho} H_{\mu^{\prime} \nu^{\prime} \rho^{\prime}}\right. \\
\left.-e^{-2 \Phi} g^{\mu \mu^{\prime}} g^{\nu \nu^{\prime}} F_{\mu \nu}^{(a)}(L M L)_{a b} F_{\mu^{\prime} \nu^{\prime}}^{(b)}+\frac{1}{8} g^{\mu \nu} \operatorname{Tr}\left(\partial_{\mu} M L \partial_{\nu} M L\right)\right]
\end{aligned}
$$

where

$$
\begin{aligned}
F_{\mu \nu}^{(a)} & =\partial_{\mu} A_{\nu}^{(a)}-\partial_{\nu} A_{\mu}^{(a)} \\
H_{\mu \nu \rho} & =\left(\partial_{\mu} B_{\nu \rho}+2 A_{\mu}^{(a)} L_{a b} F_{\nu \rho}^{(b)}\right)+\text { cyclic permutations of } \mu, \nu, \rho,
\end{aligned}
$$

and $R_{g}$ is the scalar curvature associated with the three dimensional metric $g_{\mu \nu}$. In deriving this result we have chosen the normalization constant $C$ in Eq.(2.1) in such a way that we get an overall normalization factor of 1/4 in front of Eq.(2.6).

The action given in eq.(2.6) is invariant under the $\mathrm{O}(7,23)$ transformation

$$
M \rightarrow \widetilde{\Omega} M \widetilde{\Omega}^{T}, \quad A_{\mu}^{(a)} \rightarrow \widetilde{\Omega}_{a b} A_{\mu}^{(b)}, \quad g_{\mu \nu} \rightarrow g_{\mu \nu}, \quad B_{\mu \nu} \rightarrow B_{\mu \nu}, \quad \Phi \rightarrow \Phi,
$$

where $\widetilde{\Omega}$ is an $\mathrm{O}(7,23)$ matrix, i.e. a $30 \times 30$ matrix, satisfying,

$$
\widetilde{\Omega} L \widetilde{\Omega}^{T}=L .
$$

In three dimensions the field $B_{\mu \nu}$ has no physical degrees of freedom. Using this fact, we shall restrict ourselves to backgrounds characterized by

$$
H_{\mu \nu \rho}=0
$$

The equations of motion of the gauge fields $A_{\mu}^{(a)}$ now take the form:

$$
\partial_{\mu}\left(e^{-2 \Phi} \sqrt{-g} g^{\mu \mu^{\prime}} g^{\nu \nu^{\prime}}(M L)_{a b} F_{\mu^{\prime} \nu^{\prime}}^{(b)}\right)=0
$$


This allows us to define a set of scalar fields $\psi^{a}$ through the relations

$$
\sqrt{-g} e^{-2 \Phi} g^{\mu \mu^{\prime}} g^{\nu \nu^{\prime}}(M L)_{a b} F_{\mu^{\prime} \nu^{\prime}}^{(b)}=\frac{1}{2} \epsilon^{\mu \nu \rho} \partial_{\rho} \psi^{a}
$$

The Bianchi identity for $F_{\mu \nu}^{(a)}, \epsilon^{\mu \nu \rho} \partial_{\mu} F_{\nu \rho}^{(a)}=0$, now takes the form:

$$
D^{\mu}\left(e^{2 \Phi}(M L)_{a b} \partial_{\mu} \psi^{b}\right)=0
$$

where $D_{\mu}$ denotes the covariant derivative which preserves the metric $g_{\mu \nu}$.

Let us now regard $\psi$ as a 30 dimensional column vector, and define a new $32 \times 32$ matrix $\mathcal{M}$ as follows

$\mathcal{M}=\left(\begin{array}{ccc}M+e^{2 \Phi} \psi \psi^{T} & -e^{2 \Phi} \psi & M L \psi+\frac{1}{2} e^{2 \Phi} \psi\left(\psi^{T} L \psi\right) \\ -e^{2 \Phi} \psi^{T} & e^{2 \Phi} & -\frac{1}{2} e^{2 \Phi} \psi^{T} L \psi \\ \psi^{T} L M+\frac{1}{2} e^{2 \Phi} \psi^{T}\left(\psi^{T} L \psi\right) & -\frac{1}{2} e^{2 \Phi} \psi^{T} L \psi & e^{-2 \Phi}+\psi^{T} L M L \psi+\frac{1}{4} e^{2 \Phi}\left(\psi^{T} L \psi\right)^{2}\end{array}\right)$

It can be verified by straightforward algebraic manipulations that the matrix $\mathcal{M}$ satisfies the relations

$$
\mathcal{M}^{T}=\mathcal{M}, \quad \mathcal{M}^{T} \mathcal{L} \mathcal{M}=\mathcal{L},
$$

where $\mathcal{L}$ is a $32 \times 32$ matrix

$$
\mathcal{L}=\left(\begin{array}{lll}
L & 0 & 0 \\
0 & 0 & 1 \\
0 & 1 & 0
\end{array}\right)
$$

For $H_{\mu \nu \rho}=0$, the equations of motion derived from the action (2.6), together with eq. (2.13), can be shown to be identical to the equations of motion derived from the 
action

$$
S=\frac{1}{4} \int d^{3} x \sqrt{-g}\left[R_{g}+\frac{1}{8} g^{\mu \nu} \operatorname{Tr}\left(\partial_{\mu} \mathcal{M} \mathcal{L} \partial_{\nu} \mathcal{M L}\right)\right]
$$

This action is manifestly invariant under the $\mathrm{O}(8,24)$ transformation

$$
\mathcal{M} \rightarrow \Omega \mathcal{M} \Omega^{T}, \quad g_{\mu \nu} \rightarrow g_{\mu \nu}
$$

where $\Omega$ is a $32 \times 32$ matrix satisfying,

$$
\Omega \mathcal{L} \Omega^{T}=\mathcal{L}
$$

This establishes the $\mathrm{O}(8,24)$ invariance of the low energy effective field theory describing the three dimensional string theory.

We shall now show that this $\mathrm{O}(8,24)$ symmetry of the action may be understood in terms of the $\mathrm{O}(7,23)$ symmetry $(2.8)$, and the $\mathrm{SL}(2, \mathrm{R})$ symmetry of the four dimensional effective action discussed in Ref.[6]. We regard the three dimensional effective theory as the result of dimensional reduction of the four dimensional effective theory to three dimensions. Then the three dimensional effective theory should possess all the symmetries of the four dimensional effective theory, as long as they are not broken by compactification. In particular, the $\operatorname{SL}(2, \mathrm{R})$ symmetry of the four dimensional effective action (whose discrete subgroup SL(2,Z) may be identified with the S-duality group of the full four dimensional theory) should induce an SL(2,R) symmetry of the three dimensional theory. There are many ways of regarding a three dimensional theory as a compactified four dimensional theory; let us, for definiteness, take the four dimensional theory to be the one obtained by compactifying the directions 4-9. Then the three dimensional theory is obtained by compactifying the third direction of the four dimensional theory on a circle. The action of the $\mathrm{SL}(2, \mathrm{R})$ group on various 'four dimensional fields' has been given in Ref.[6]. From this one can find the transformation laws of various ten dimensional fields, and hence, by using eq.(2.3), (2.12) and (2.14), the transformation laws of 
various 'three dimensional fields'. It turns out that the $\mathrm{SL}(2, \mathrm{R})$ transformation of the 'four dimensional fields' $-\lambda \rightarrow(a \lambda+b) /(c \lambda+d)$ - generated by the matrix $\left(\begin{array}{ll}a & b \\ c & d\end{array}\right)$ with $a d-b c=1$, corresponds to the following transformation on the 'three dimensional fields':

$$
\mathcal{M} \rightarrow \Omega \mathcal{M} \Omega^{T}
$$

where,

$$
\Omega=\left(\begin{array}{ccccccc}
a & 0 & 0 & 0 & 0 & b & 0 \\
0 & I_{6} & 0 & 0 & 0 & 0 & 0 \\
0 & 0 & d & 0 & 0 & 0 & -c \\
0 & 0 & 0 & I_{6} & 0 & 0 & 0 \\
0 & 0 & 0 & 0 & I_{16} & 0 & 0 \\
c & 0 & 0 & 0 & 0 & d & 0 \\
0 & 0 & -b & 0 & 0 & 0 & a
\end{array}\right)
$$

It can be easily verified that $\Omega$ given above represents an $\mathrm{O}(8,24)$ transformation. Furthermore, one can also verify that the full $\mathrm{O}(8,24)$ group of transformations may be generated as a combination of the $\mathrm{O}(7,23)$ transformation $(2.8)$, and the $\mathrm{SL}(2, \mathrm{R})$ transformation given above. Thus the $\mathrm{O}(8,24)$ symmetry of the three dimensional effective action may be regarded as a consequence of the $\mathrm{O}(7,23)$ symmetry, and the SL(2,R) symmetry of the four dimensional theory.

We shall argue in the next section that an $\mathrm{O}(8,24 ; \mathrm{Z})$ subgroup of this is a symmetry of the full string theory. In doing so, we must first identify an appropriate $\mathrm{O}(8,24 ; \mathrm{Z})$ subgroup of the full $\mathrm{O}(8,24)$ group which we want to show is the symmetry of the full theory. ${ }^{\star}$ Let us first identify the $\mathrm{O}(7,23 ; \mathrm{Z})$ subgroup of the $\mathrm{O}(8,24)$ that represents the usual target space duality symmetry of the three dimensional

\footnotetext{
* In the basis that we have chosen, $\mathrm{O}(8,24 ; \mathrm{Z})$ matrices are not $\mathrm{O}(8,24)$ matrices with integer entries.
} 
string theory [21]. This subgroup is generated by matrices of the form:

$$
\left(\begin{array}{lll}
\widetilde{\Omega} & 0 & 0 \\
0 & 1 & 0 \\
0 & 0 & 1
\end{array}\right),
$$

where $\widetilde{\Omega}$ is an $\mathrm{O}(7,23)$ matrix satisfying the condition that $L \widetilde{\Omega} L$ preserves the 30 dimensional Lorenzian, even, self-dual lattice $\Lambda_{30}$, with metric $L$, which represents the allowed values of the electric charge vectors of elementary string states [28]. This specifies the $\mathrm{O}(7,23 ; \mathrm{Z})$ subgroup of $\mathrm{O}(8,24)$ completely. For convenience, we shall choose the lattice $\Lambda_{30}$ as the lattice containing the set of vectors

$$
\left(\begin{array}{c}
n_{1} \\
\cdot \\
\cdot \\
n_{14} \\
\vec{\xi}
\end{array}\right), \quad n_{i} \in Z, \quad \vec{\xi} \in \Lambda_{E_{8} \times E_{8}}
$$

where $\Lambda_{E_{8} \times E_{8}}$ is the root lattice of $E_{8} \times E_{8}$. This means that in the absence of any background scalar field, the theory represents the $E_{8} \times E_{8}$ heterotic string theory, compactified on a seven torus, with each of the seven radii being equal to the self-dual radius. In the normalization convention of ref.[6] $\alpha^{\prime}=16$, and hence the self-dual radius corresponds to $R=\sqrt{\alpha^{\prime}}=4$. This also shows that the normalization factor of $1 / 4$ in eq.(2.17) is consistent with the normalization factor of $1 / 32 \pi$ that appears in front of the four dimensional effective action of ref.[6], with the extra factor of $8 \pi$ coming from the length of the extra internal dimension. The compatibility of the normalizations of the four and the three dimensional effective actions will be important for us in our later analysis, since we shall use the known solitons in four dimensions to construct three dimensional solitons. Note that by restricting the lattice $\Lambda_{30}$ in this way we do not suffer from any loss of generality, since any other choice of the lattice is equivalent to the lattice $\Lambda_{30}$ described above, with an appropriate choice of the background scalar fields [28] [29]. 
We shall now specify the $\mathrm{O}(8,24 ; \mathrm{Z})$ subgroup of $\mathrm{O}(8,24)$ by prescribing a 32 dimensional lattice $\Lambda_{32}$ which is preserved by the $\mathrm{O}(8,24 ; \mathrm{Z})$ transformations. We take this lattice to be the collection of vectors of the form:

$$
\left(\begin{array}{c}
\vec{\beta} \\
m_{1} \\
m_{2}
\end{array}\right), \quad \vec{\beta} \in \Lambda_{30}, \quad m_{1}, m_{2} \in Z .
$$

$\mathrm{O}(8,24 ; \mathrm{Z})$ subgroup of $\mathrm{O}(8,24)$ is defined to be the group of $\mathrm{O}(8,24)$ matrices $\Omega$, satisfying the condition that $\mathcal{L} \Omega \mathcal{L}$ preserves the lattice $\Lambda_{32}$. (Equivalently, $\Omega$ preserves the lattice $\mathcal{L} \Lambda_{32}$.) In the next section we shall discuss the possibility that this $\mathrm{O}(8,24 ; \mathrm{Z})$ subgroup is a symmetry of the full string theory.

Using eqs.(2.23) and (2.24) we see that the lattice $\Lambda_{32}$ contains the set of vectors

$$
\left(\begin{array}{c}
n_{1} \\
\cdot \\
\cdot \\
n_{14} \\
\vec{\xi} \\
m_{1} \\
m_{2}
\end{array}\right), \quad n_{i}, m_{i} \in Z, \quad \vec{\xi} \in \Lambda_{E_{8} \times E_{8} .}
$$

In order to check that we are on the right track, we shall now verify that the Sduality group $\mathrm{SL}(2, \mathrm{Z})$ of the four dimensional theory is a subgroup of the $\mathrm{O}(8,24 ; \mathrm{Z})$ group that preserves the above lattice. To do this, we regard the 3-dimensional theory as a four dimensional theory compactified on a circle as before, taking the four dimensional theory to be the one obtained by compactifying the directions 4-9. From eqs.(2.20), (2.21), we see that the $\mathrm{SL}(2, \mathrm{Z})$ transformation generated by the matrix $\left(\begin{array}{ll}p & q \\ r & s\end{array}\right)$ with $p s-q r=1, p, q, r, s \in Z$, corresponds to an $\mathrm{O}(8,24)$ 
transformation generated by:

$$
\Omega=\left(\begin{array}{ccccccc}
p & 0 & 0 & 0 & 0 & q & 0 \\
0 & I_{6} & 0 & 0 & 0 & 0 & 0 \\
0 & 0 & s & 0 & 0 & 0 & -r \\
0 & 0 & 0 & I_{6} & 0 & 0 & 0 \\
0 & 0 & 0 & 0 & I_{16} & 0 & 0 \\
r & 0 & 0 & 0 & 0 & s & 0 \\
0 & 0 & -q & 0 & 0 & 0 & p
\end{array}\right) .
$$

It can be easily verified that $\Omega$ given above preserves the lattice $\mathcal{L} \Lambda_{32}$ for $\Lambda_{32}$ defined by eq.(2.25), and hence represents an $\mathrm{O}(8,24 ; \mathrm{Z})$ transformation. If, instead of regarding the 3 direction as one of the four dimensions, we choose any of the other directions $4-9$ as one of the four dimensions, the matrix $\Omega$ is modified appropriately, but in each case represents an $\mathrm{O}(8,24 ; \mathrm{Z})$ transformation.

The three dimensional string theory is manifestly $\mathrm{O}(7,23 ; \mathrm{Z})$ invariant. Thus establishing $\mathrm{O}(8,24 ; \mathrm{Z})$ invariance of the theory basically amounts to showing that the original $\mathrm{SL}(2, \mathrm{Z})$ symmetry of the four dimensional theory is not destroyed when we compactify one of the three space-like directions in this theory. In the next two sections we shall try to identify some of the soliton states in the string theory which are related to the elementary string excitations via the above $\mathrm{SL}(2, \mathrm{Z})$ subgroup of $\mathrm{O}(8,24 ; \mathrm{Z})$ transformations. The existence of these soliton states is a necessary requirement for the $\mathrm{O}(8,24 ; \mathrm{Z})$ invariance of the theory.

\footnotetext{
$\star$ Recent results of ref.[9] provide strong evidence in favor of this.
} 


\section{Elementary Particles and Solitons}

The charge quantum numbers of elementary string excitations are characterized by a 30 dimensional vector $\vec{\alpha} \in \Lambda_{30}$. The asymptotic value of the field strength $F_{\mu \nu}^{(a)}$ associated with such an elementary particle can be calculated using the procedure outlined in ref.[6]. The answer in polar coordinates is

$$
\sqrt{-g} F^{(a) 0 \rho} \simeq-\frac{1}{4 \pi} e^{2 \Phi} M_{a b} \alpha^{b}
$$

where $(\rho, \theta)$ are the polar coordinates of the two dimensional space, with origin at the location of the elementary particle. Using eq.(2.12), we get the following asymptotic form of $\psi^{a}$ :

$$
\psi^{a} \simeq-\frac{\theta}{2 \pi} L_{a b} \alpha^{b}+\text { constant }
$$

Thus under $\theta \rightarrow \theta-2 \pi, \psi^{a} \rightarrow \psi^{a}+L_{a b} \alpha^{b}$. This induces the following transformation of the matrix $\mathcal{M}$ :

$$
\mathcal{M} \rightarrow \Omega_{E}(\vec{\alpha}) \mathcal{M} \Omega_{E}^{T}(\vec{\alpha})
$$

where,

$$
\Omega_{E}(\vec{\alpha})=\left(\begin{array}{ccc}
I_{30} & -L \alpha & 0 \\
0 & 1 & 0 \\
\alpha^{T} & -\frac{1}{2} \vec{\alpha}^{2} & 1
\end{array}\right), \quad \vec{\alpha}^{2} \equiv \alpha^{T} L \alpha .
$$

One can easily verify that $\Omega_{E}(\vec{\alpha})$ is an $\mathrm{O}(8,24)$ matrix. Furthermore, since the lattice $\Lambda_{30}$ is even and self-dual with respect to the metric $L$, we have $\vec{\alpha}^{2} \equiv$ $\alpha^{T} L \alpha=$ even, and $\alpha^{T} L \beta=$ integer for $\vec{\alpha}, \vec{\beta} \in \Lambda_{30}$. Using these relations one can verify that $\mathcal{L} \Omega_{E}(\vec{\alpha}) \mathcal{L}$ preserves the lattice $\Lambda_{32}$, i.e. acting on a vector of the form given in eq.(2.24), it produces another vector of the same form. Thus $\Omega_{E}(\vec{\alpha}) \in$ $O(8,24 ; Z)$. This shows that elementary string excitations in three dimensions may be regarded as vortices, with the matrix valued field $\mathcal{M}$ transforming by an $\mathrm{O}(8,24 ; \mathrm{Z})$ transformation as we go around the vortex. 
Of special interest are the elementary string excitations which are invariant under half of the supersymmetry transformations, and hence saturate the Bogomol'nyi bound [13]. These were analyzed in detail in refs.[6][8]. Elementary string states with $\vec{\alpha}^{2}=-2$ and saturating the Bogomol'nyi bound are 16 fold degenerate, whereas those with $\vec{\alpha}^{2}=0$ and saturating the Bogomol'nyi bound are $16 \times 24$ fold degenerate.

At this stage we should point out that due to infra-red logarithmic divergence in the electro-magnetic contribution to the self energy, both the elementary string excitations and the soliton masses are divergent in this theory. This does not contradict the Bogomol'nyi formula, since the asymptotic values of the scalar fields which appear in the expression for the Bogomol'nyi bound, also diverge logarithmically. Because of the divergent self-energies, it is more convenient to use the criteria of unbroken supersymmetry rather than saturation of the Bogomol'nyi bound to characterize these states, although we shall continue to refer to them as states saturating the Bogomol'nyi bound. The presence of these infra-red divergences puts the analysis in the three dimensional theory on a less solid footing than the corresponding analysis in the four dimensional theory. However, these infrared divergences affect the elementary particles and solitons in an identical manner since they are caused by massless scalars and abelian gauge fields, and, as we have seen in sec.2, the dynamics of these fields is manifestly $\mathrm{O}(8,24 ; \mathrm{Z})$ symmetric. Thus, whatever be the physical effect of these infra-red divergences, we do not expect them to cause a violation of the $\mathrm{O}(8,24 ; \mathrm{Z})$ symmetry. In view of this, we shall continue to analyze the spectrum of states in this theory ignoring the infra-red divergences. It will also be understood that when we relate the asymptotic behavior of various fields with various charge quantum numbers, we shall be talking of an asymptotic region where the logarithmic growth of various fields have not reached a large enough value so as to invalidate the classical analysis.

Let us now turn to the analysis of the non-singular soliton solutions in the three dimensional string theory. A class of these solutions may be constructed using an idea developed in ref.[30], namely by starting from a periodic array of 
known monopole solutions in four dimensions along one particular direction (say the 3 direction) with periodicity $2 \pi R_{(3)}$, and reinterpreting it as a three dimensional solution, with the third direction compactified on a circle of radius $R_{(3)}$. (As stated before, here $R_{(3)}=4$.) There are two classes of known non-singular monopole solutions in four dimensions, the BPS monopole and the $H$-monopole, and in both cases multiple monopole solutions are known to exist. SL(2,Z) invariance of the four dimensional theory in fact demands the existence of many other multi-monopole solutions, with each monopole carrying magnetic charge vector $\bar{\beta}$, for some specific set of vectors $\bar{\beta}$ belonging to a 28-dimensional self-dual Lorentzian lattice [6]. In our analysis of this section we shall assume the existence of solutions representing periodic arrays of these monopoles; we shall not, however, need to know the specific forms of these solutions. Quantization of these soliton solutions will be discussed only for the periodic arrays of BPS monopole solutions, since this is the case that is best understood. For this purpose, we have discussed some of the properties of these solutions in appendices A-C. (Quantization of even a single $H$-monopole solution is plagued by our lack of understanding of the physics at large and small distance scales [8], although the classical multiple $H$-monopole solutions can be constructed explicitly [30].)

Let us consider a magnetic monopole solution in four dimensions carrying magnetic charge vector $\bar{\beta}$. $\quad\left(\bar{\beta}^{2}=-2\right.$ corresponds to BPS monopoles, and $\bar{\beta}^{2}=0$ corresponds to $H$-monopole solutions.) If $\bar{A}_{\bar{\mu}}^{(\bar{a})}$ denotes the four dimensional vector fields $(1 \leq \bar{a} \leq 28,0 \leq \bar{\mu} \leq 3)$, then the total magnetic flux of the gauge field $\bar{A}_{\bar{\mu}}^{(\bar{a})}$ associated with this monopole solution is given by $4 \pi \bar{L}_{\bar{a} \bar{b}} \bar{\beta}^{\bar{b}}$ [6], where,

$$
\bar{L}=\left(\begin{array}{ccc}
0 & I_{6} & 0 \\
I_{6} & 0 & 0 \\
0 & 0 & -I_{16}
\end{array}\right)
$$

Let us now consider an array of these monopoles in the 3 direction separated by a distance $8 \pi$ (which corresponds to a radius of compactification 4 , the self-dual

\footnotetext{
$\star$ In fact, construction of many of these solutions may require us to take stringy effects into account.
} 
radius). Then the total flux per unit length coming out of a cylinder enclosing the 3 axis will be $\frac{1}{2} \bar{L}_{\bar{a} \bar{b}} \bar{\beta}^{\bar{b}}$. As has been argued in appendix A, asymptotically, the dependence of various fields on the third direction can be ignored. Thus if $(\rho, \theta)$ denote the polar coordinates of the 2 dimensional space spanned by the 1,2 directions, we get,

$$
\partial_{\theta} \bar{A}_{3}^{(\bar{a})} \simeq-\frac{1}{4 \pi} \bar{L}_{\bar{a} \bar{b}} \bar{\beta}^{\bar{b}}
$$

Thus $\bar{A}_{3}^{(\bar{a})}$ increases by $\frac{1}{2} \bar{L}_{\bar{a} \bar{b}} \bar{\beta}^{\bar{b}}$ as $\theta \rightarrow \theta-2 \pi$.

The relationship between four dimensional fields and the ten dimensional fields was given in ref.[6]. Hence from eq.(3.6) we can determine how the asymptotic values of different ten dimensional fields transform under $\theta \rightarrow \theta-2 \pi$. This, in turn, can be used to calculate how the asymptotic values of different three dimensional fields, defined through eq.(2.3), (2.4), (2.12) and (2.14), transform under $\theta \rightarrow \theta-2 \pi$. We find that if we identify the direction $\bar{\mu}=3$ with the first compact direction of the 7 dimensional torus $(m=1)$, and if,

$$
\bar{\beta}=-\left(\begin{array}{c}
\vec{a}_{1} \\
\vec{a}_{2} \\
\vec{\xi}
\end{array}\right), \quad \vec{a}_{1}, \vec{a}_{2} \in Z^{6}, \quad \vec{\xi} \in \Lambda_{E_{8} \times E_{8}}
$$

then, as $\theta \rightarrow \theta-2 \pi$,

$$
\mathcal{M} \rightarrow \Omega_{S}\left(\vec{a}_{1}, \vec{a}_{2}, \vec{\xi}\right) \mathcal{M} \Omega_{S}^{T}\left(\vec{a}_{1}, \vec{a}_{2}, \vec{\xi}\right),
$$

where,

$$
\Omega_{S}\left(\vec{a}_{1}, \vec{a}_{2}, \vec{\xi}\right)=\left(\begin{array}{ccccccc}
1 & 0 & 0 & 0 & 0 & 0 & 0 \\
a_{2} & I_{6} & 0 & 0 & 0 & 0 & 0 \\
-\frac{1}{2} \bar{\beta}^{2} & -a_{1}^{T} & 1 & -a_{2}^{T} & -\xi^{T} & 0 & 0 \\
a_{1} & 0 & 0 & I_{6} & 0 & 0 & 0 \\
-\xi & 0 & 0 & 0 & I_{16} & 0 & 0 \\
0 & 0 & 0 & 0 & 0 & 1 & 0 \\
0 & 0 & 0 & 0 & 0 & 0 & 1
\end{array}\right) .
$$


Here,

$$
\bar{\beta}^{2} \equiv \bar{\beta}^{T} \bar{L} \bar{\beta}=2 \vec{a}_{1} \cdot \vec{a}_{2}-\vec{\xi}^{2}
$$

It can be easily verified that $\Omega_{S}\left(\vec{a}_{1}, \vec{a}_{2}, \vec{\xi}\right)$ is an $\mathrm{O}(8,24 ; \mathrm{Z})$ matrix. Thus we see that these soliton solutions can also be regarded as vortex solutions, with the matrix $\mathcal{M}$ transforming by a non-trivial $\mathrm{O}(8,24 ; \mathrm{Z})$ transformation as we go around the vortex. In fact, $\Omega_{S}$ represents an element of the target space duality group $\mathrm{O}(7,23 ; \mathrm{Z})$. Although, unlike ordinary vortex solutions, these solutions depend on the internal coordinate $x^{3}$, it has been argued in appendix A that the $x^{3}$ dependence of various fields falls off exponentially as we move away from the soliton core.

We shall shortly see that the elementary particles and the soliton solutions are related by $\mathrm{O}(8,24 ; \mathrm{Z})$ transformations. However, we shall first generalize the soliton solution by taking into account the electric charge quantum numbers that the soliton is allowed to carry. First of all, the four dimensional monopole solution can carry an electric charge vector $-n \bar{\beta}$ for any integer $n$, this electric charge arises from the quantization of an appropriate zero mode of the monopole solution. This induces asymptotic values of $\partial_{\theta} \psi^{a}$ as in the case of electrically charged elementary string excitations. Furthermore, the solution can also carry a momentum in the internal direction labelled by $\bar{\mu}=3$. This momentum will be labelled by an integer $k$, and, from the three dimensional viewpoint, will correspond to a new charge quantum number associated with the three dimensional gauge fields. Hence this will also induce an asymptotic value of $\partial_{\theta} \psi^{a}$. The net result is that for a three dimensional soliton labelled by the quantum numbers $(\bar{\beta}, n, k)$,

$$
\mathcal{M} \rightarrow \Omega_{S}\left(\vec{a}_{1}, \vec{a}_{2}, \vec{\xi} ; n, k\right) \mathcal{M} \Omega_{S}^{T}\left(\vec{a}_{1}, \vec{a}_{2}, \vec{\xi} ; n, k\right), \quad \text { as } \theta \rightarrow \theta-2 \pi
$$

where, 


$$
\Omega_{S}\left(\vec{a}_{1}, \vec{a}_{2}, \vec{\xi} ; n, k\right)=\left(\begin{array}{ccccccc}
1 & 0 & 0 & 0 & 0 & 0 & 0 \\
a_{2} & I_{6} & 0 & 0 & 0 & -n a_{2} & 0 \\
-\frac{1}{2} \bar{\beta}^{2} & -a_{1}^{T} & 1 & -a_{2}^{T} & -\xi^{T} & \frac{1}{2} n \bar{\beta}^{2}-k & 0 \\
a_{1} & 0 & 0 & I_{6} & 0 & -n a_{1} & 0 \\
-\xi & 0 & 0 & 0 & I_{16} & n \xi & 0 \\
0 & 0 & 0 & 0 & 0 & 1 & 0 \\
\frac{n}{2} \bar{\beta}^{2}+k & n a_{1}^{T} & 0 & n a_{2}^{T} & n \xi^{T} & -\frac{1}{2} n^{2} \bar{\beta}^{2} & 1
\end{array}\right)
$$

It can be verified that $\Omega_{S}\left(\vec{a}_{1}, \vec{a}_{2}, \vec{\xi} ; n, k\right)$ also represents an $\mathrm{O}(8,24 ; \mathrm{Z})$ matrix.

Thus we see that both, the elementary string excitations, and solitonic states in the theory, saturating the Bogomol'nyi bound, are characterized by $\mathrm{O}(8,24 ; \mathrm{Z})$ matrices $\Omega$, such that the matrix $\mathcal{M}$ transforms to $\Omega \mathcal{M} \Omega^{T}$ as we go around the state once. Let us now consider the effect of an $\mathrm{O}(8,24 ; \mathrm{Z})$ transformation $\omega$ on such a state. Under such a transformation,

$$
\mathcal{M} \rightarrow \mathcal{M}^{\prime}=\omega \mathcal{M} \omega^{T}
$$

Thus, as $\theta \rightarrow \theta-2 \pi$,

$$
\mathcal{M}^{\prime}=\omega \mathcal{M} \omega^{T} \rightarrow \omega \Omega \mathcal{M} \Omega^{T} \omega^{T}=\omega \Omega \omega^{-1} \mathcal{M}^{\prime}\left(\omega^{T}\right)^{-1} \Omega^{T} \omega^{T}=\Omega^{\prime} \mathcal{M}^{\prime} \Omega^{\prime T}
$$

where,

$$
\Omega^{\prime}=\omega \Omega \omega^{-1}
$$

Thus under an $\mathrm{O}(8,24 ; \mathrm{Z})$ transformation generated by $\omega$, a state characterized by the matrix $\Omega$ goes to a state characterized by the matrix $\Omega^{\prime}=\omega \Omega \omega^{-1}$.

We shall now show that the solitons characterized by the matrices $\Omega_{S}\left(\vec{a}_{1}, \vec{a}_{2}, \vec{\xi} ; n, k\right)$ given in eq.(3.12) are related to the elementary string excitations characterized by the matrix $\Omega_{E}(\vec{\alpha})$ given in eq.(3.4) through an $\mathrm{O}(8,24 ; \mathrm{Z})$ transformation. This is 
easily verified by noting that

$$
\Omega_{S}\left(\vec{a}_{1}, \vec{a}_{2}, \vec{\xi} ; n, k\right)=\omega_{n} \Omega_{E}\left(\vec{\alpha}_{0}\right) \omega_{n}^{-1}
$$

where,

$$
\vec{\alpha}_{0}=\left(\begin{array}{c}
k \\
\vec{a}_{1} \\
0 \\
\vec{a}_{2} \\
\vec{\xi}
\end{array}\right), \quad \vec{\alpha}_{0}^{2} \equiv \alpha_{0}^{T} L \alpha_{0}=2 \vec{a}_{1} \cdot \vec{a}_{2}-\xi^{2}
$$

and,

$$
\omega_{n}=\left(\begin{array}{ccccccc}
n & 0 & 0 & 0 & 0 & -1 & 0 \\
0 & I_{6} & 0 & 0 & 0 & 0 & 0 \\
0 & 0 & 0 & 0 & 0 & 0 & -1 \\
0 & 0 & 0 & I_{6} & 0 & 0 & 0 \\
0 & 0 & 0 & 0 & I_{16} & 0 & 0 \\
1 & 0 & 0 & 0 & 0 & 0 & 0 \\
0 & 0 & 1 & 0 & 0 & 0 & n
\end{array}\right)
$$

This shows that the soliton solutions are related to the elementary excitations in string theory via an $\mathrm{O}(8,24 ; \mathrm{Z})$ transformation. ${ }^{\star}$ Note that in constructing the soliton solutions we have treated the internal direction $m=1$ as special, since that is the direction we have identified as one of the directions in the four dimensional theory. Other soliton solutions in the theory, corresponding to any other internal direction being identified as the fourth direction may be constructed in a similar manner. These solitons will also be related to the elementary string excitations by appropriate $\mathrm{O}(8,24 ; \mathrm{Z})$ transformation.

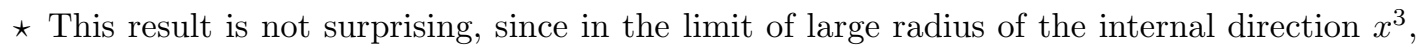
both the elementary particles and the solitons can be identified to the corresponding states in four dimensions, which are known to be related by $\mathrm{SL}(2, \mathrm{Z})$ transformations. But as we shall see in the next section, $\mathrm{O}(8,24 ; \mathrm{Z})$ symmetry makes non-trivial predictions about the moduli space of multi-soliton solutions. 
So far we have only shown that the quantum numbers of the solitons are related to those of elementary particles via an $\mathrm{O}(8,24 ; \mathrm{Z})$ transformation. However, $\mathrm{O}(8,24 ; \mathrm{Z})$ invariance also requires that the degeneracy of states is identical for the elementary particles and solitons. In order to investigate this question, we need to quantize the collective coordinates of the soliton solutions. As mentioned at the beginning of this discussion, this will be done only for the periodic arrays of BPS monopole solutions (characterized by $\bar{\beta}^{2}=-2$ ). From the relationship between $\vec{\alpha}_{0}$ and $\bar{\beta}$ given in eqs.(3.7), (3.17) it follows that the corresponding elementary string states have $\left(\vec{\alpha}_{0}\right)^{2}=-2$, and hence are 16 fold degenerate [6]. On the other hand, the moduli space of periodic array of single BPS monopoles is 4 dimensional, and is given by $R^{2} \times S^{1} \times S^{1}$, with $R^{2}$ representing the location of the array in the non-compact directions, the first $S^{1}$ denoting the location of the array in the compact direction, and the second $S^{1}$ representing the coordinate conjugate to the $\mathrm{U}(1)$ electric charge. ${ }^{\dagger}$ The quantum numbers $k$ and $n$ can be identified to momenta conjugate to these two angular coordinates. The structure of the fermionic zero modes is identical to that of a single BPS monopole in 4 dimensions with moduli space $R^{3} \times S^{1}$, since the supersymmetry algebra, as well as the number of unbroken supersymmetry generators is the same in the two cases. Standard analysis [2] [32] then shows that the soliton state is 16-fold degenerate, in agreement with the requirement of $\mathrm{O}(8,24 ; \mathrm{Z})$ symmetry.

In our discussion relating the solitons to elementary excitations, we have considered only those elementary string states which do not carry any winding number in the $x^{3}(m=1)$ direction. This can be seen from eq.(3.17). Strings winding along the $x^{3}$ direction will play a crucial role in the analysis of section 5 .

$\dagger$ As is typical for solitons in three dimensions [31], the zero modes associated with $R^{2} \times S^{1} \times S^{1}$ are not strictly normalizable due to a logarithmic divergence from large distances. This divergence is related to the fact that these solitons have infinite mass due to logarithmic divergence in the integral of energy density from large distance scale, and also affects the elementary string states. A possible infra-red regularization that gives rise to normalizable zero modes associated with the $R^{2} \times S^{1} \times S^{1}$ factor has been discussed in appendix C. 


\section{Predictions of $\mathrm{O}(8,24 ; \mathrm{Z})$ Invariance}

As in the case of four dimensional theory, existence of $\mathrm{O}(8,24 ; \mathrm{Z})$ symmetry predicts the existence of many new soliton states in the theory. We shall consider only some specific examples here. Let us consider the $\mathrm{O}(8,24 ; \mathrm{Z})$ matrix

$$
\omega=\left(\begin{array}{ccccccc}
p & 0 & 0 & 0 & 0 & q & 0 \\
0 & I_{6} & 0 & 0 & 0 & 0 & 0 \\
0 & 0 & s & 0 & 0 & 0 & -r \\
0 & 0 & 0 & I_{6} & 0 & 0 & 0 \\
0 & 0 & 0 & 0 & I_{16} & 0 & 0 \\
r & 0 & 0 & 0 & 0 & s & 0 \\
0 & 0 & -q & 0 & 0 & 0 & p
\end{array}\right), \quad p s-q r=1 .
$$

Applying this $\mathrm{O}(8,24 ; \mathrm{Z})$ transformation on an elementary string excitation characterized by the matrix $\Omega_{E}\left(\vec{\alpha}_{0}\right)$, with $\vec{\alpha}_{0}$ given by eq.(3.17), we get the following $\mathrm{O}(8,24 ; \mathrm{Z})$ matrix:

$$
\omega \Omega_{E}\left(\vec{\alpha}_{0}\right) \omega^{-1}=\left(\begin{array}{ccccccc}
1 & 0 & 0 & 0 & 0 & 0 & 0 \\
r a_{2} & I_{6} & 0 & 0 & 0 & -p a_{2} & 0 \\
-\frac{r^{2}}{2} \vec{\alpha}_{0}^{2} & -r a_{1}^{T} & 1 & -r a_{2}^{T} & -r \xi^{T} & -k+\frac{p r}{2} \vec{\alpha}_{0}^{2} & 0 \\
r a_{1} & 0 & 0 & I_{6} & 0 & -p a_{1} & 0 \\
-r \xi & 0 & 0 & 0 & I_{16} & p \xi & 0 \\
0 & 0 & 0 & 0 & 0 & 1 & 0 \\
k+\frac{p r}{2} \vec{\alpha}_{0}^{2} & p a_{1}^{T} & 0 & p a_{2}^{T} & p \xi^{T} & -\frac{p^{2}}{2} \vec{\alpha}_{0}^{2} & 1
\end{array}\right)
$$

Reversing the analysis that led to eq.(3.12), we can easily verify that a state characterized by the above $\mathrm{O}(8,24 ; \mathrm{Z})$ matrix corresponds to a periodic array of $r$ monopoles in four dimensions, carrying electric and magnetic charge quantum numbers $-p \bar{\beta}$ and $r \bar{\beta}$ respectively, and carrying $k$ units of momentum in the internal direction $\bar{\mu}=3$. For $\bar{\beta}^{2}=-2$, i.e. for BPS monopoles, $\mathrm{O}(8,24 ; \mathrm{Z})$ invariance again predicts a 16 fold degeneracy of these states. 
In order to verify this prediction, one may proceed as follows. First one assumes that the scale of breaking of the $\mathrm{SU}(2)$ symmetry that leads to the construction of the BPS monopoles, as well as the inverse radius of the third direction, is small compared to the Planck mass. In this limit we can ignore the gravitational effects, and regard the solitons as periodic array of monopole solutions in $N=4$ supersymmetric $\mathrm{SU}(2)$ gauge theories in four dimensions, with the $\mathrm{SU}(2)$ symmetry spontaneously broken by the vacuum expectation value of the Higgs field. In particular, in this limit the off-diagonal components $G_{3 \mu}^{(10)}$ of the gravitational field, which would behave like a gauge field in three dimensions and would couple to the charge quantum number $k$, is ignored, since the corresponding gauge coupling constant is proportional to the inverse radius of the third direction measured in Planck units. The question of existence of states characterized by eq.(4.2) now becomes the question of whether the $\mathrm{SL}(2, \mathrm{Z})$ symmetry of the $\mathrm{N}=4$ supersymmetric Yang-Mills theory in four dimensions survives under the compactification of one of the space directions. Although the recent results of Vafa and Witten [9] show that the $\mathrm{SL}(2, \mathrm{Z})$ invariance of the theory does not depend on the underlying four manifold, it will be reassuring to directly verify the existence of these states. We shall now discuss how this might be done.

As in the case of multi-monopole solutions, the existence of supersymmetric states representing multiple periodic arrays of monopoles, and carrying multiple units of electric charge and momentum in the 3 direction, can be studied by quantizing the collective coordinates parametrizing the moduli space of these solutions. The moduli space of $r$ BPS monopoles arranged in a periodic array has been discussed in some detail in appendix B. As explained there, this moduli space is expected to have the structure ${ }^{\star}$

$$
\mathcal{M}_{r}=R^{2} \times\left(S^{1} \times S^{1} \times \mathcal{M}_{r}^{0} / Z_{r} \times Z_{r}\right)
$$

\footnotetext{
* It has been shown in appendix $\mathrm{C}$ that even though the zero modes associated with the coordinates on $R^{2} \times S^{1} \times S^{1}$ are not normalizable, the zero modes associated with the coordinates on $\mathcal{M}_{r}^{0}$ are renormalizable.
} 
where $\mathcal{M}_{r}^{0}$ is a $4(r-1)$ dimensional manifold. The first $Z_{r}$ acts on the coordinate $X^{3}$ of the first $S^{1}$ as $X^{3} \rightarrow X^{3}-(2 \pi / r)$, and the second $Z_{r}$ acts on the coordinate $\Theta$ of the second $S^{1}$ as $\Theta \rightarrow \Theta-(2 \pi / r)$. Both the $Z_{r}$ transformations also have non-trivial action on $\mathcal{M}_{r}^{0}$. Physically, for well separated arrays, the action of the first $Z_{r}$ on $\mathcal{M}_{r}$ corresponds to translating one of the periodic arrays by one period, whereas the action of the second $Z_{r}$ on $\mathcal{M}_{r}$ corresponds to changing the $\mathrm{U}(1)$ phase of all the monopoles in one array by $2 \pi$. Since $p$ and $k$ are the quantum numbers representing the total $\mathrm{U}(1)$ charge, and the total momentum in the third direction respectively, for a given value of $p$ and $k$ the $X^{3}$ and $\Theta$ dependent part of the wave-function has the form $\exp \left(i k X^{3}+i p \Theta\right)$. Hence this part of the wave-function picks up a factor of $\exp (-2 \pi i k / r)$ under the first $Z_{r}$ transformation, and a factor of $\exp (-2 \pi i p / r)$ under the second $Z_{r}$ transformation. Thus the part of the wavefunction coming from $\mathcal{M}_{r}^{0}$ must pick up a factor of $\exp (2 \pi i k / r)$ under the action of the first $Z_{r}$ and a factor of $\exp (2 \pi i p / r)$ under the action of the second $Z_{r}$.

As was shown in ref.[33], and discussed in the specific context of monopoles in refs.[32][34][7], the supersymmetric states of the soliton, saturating the Bogomol'nyi bound, are represented by harmonic forms on the space $\mathcal{M}_{r}^{0}$. For each such harmonic form, we get a 16 fold degenerate state from quantization of the supersymmetric partners of the coordinates on $R^{2} \times S^{1} \times S^{1}$. From the relation $p s-q r=1$ it follows that the numbers $p$ and $r$ are relatively prime, but there is no restriction on $k$. Thus if we want a 16 fold degenerate state for each of these values of $p$ and $k$, we must demand that,

For every integer $p$ that is relatively prime with respect to $r$, and for every integer $k$, the space $\mathcal{M}_{r}^{0}$ has a unique (and hence (anti-)self-dual) harmonic form, which picks up a phase $\exp (2 \pi i k / r)$ under the action of the first $Z_{r}$ group, and a phase $\exp (2 \pi i p / r)$ under the action of the second $Z_{r}$ group.

At present we do not have a direct proof of the above conjecture for values of $r \geq 2$. We shall now give an indirect argument for the existence of these harmonic forms starting from the corresponding results on the $r$-monopole moduli 
space [35] [7] [10]. Before we proceed we should, however, warn the reader that this argument is based on various assumptions that look physically plausible, but it does not constitute a rigorous proof. For simplicity, we shall consider the case $r=2$, the extension to the more general case is straightforward. Let us consider the case when the radius of compactification $R_{(3)}$ of the third direction is much larger than the scale of breaking of the $\mathrm{SU}(2)$ symmetry (which we shall denote by $K)$. Let $\mathcal{R}$ denote the region of $\mathcal{M}_{2}^{0}$ where two monopoles are within a distance of order $K$ of each other, and the resulting configuration is repeated along the third direction with periodicity $2 \pi R_{(3)}$. Let us, for definiteness, assume that the 2 -monopole clusters are located around the points $2 \pi n R_{(3)}$ on the 3 -axis. In this region, the metric on $\mathcal{M}_{2}^{0}$ is close to the metric on the two monopole relative moduli space $M_{2}^{0}$, since for an infinitesimal motion in $\mathcal{M}_{2}^{0}$, the change in various fields near the monopoles in one cluster comes mostly due to the monopoles in that cluster. Contribution from the other clusters are suppressed by inverse powers of $R_{(3)}$. Now, $M_{2}^{0}$ is known [35] [7] [10] to have a self-dual harmonic form $\Omega$ which picks up a phase of -1 under the $Z_{2}$ action associated with the $U(1)$ phase. $\Omega$ falls off exponentially as the two monopoles are pulled away from each other to a distance much larger than $K$. This allows us to construct an approximate harmonic 2-form $\widehat{\Omega}$ on $\mathcal{M}_{2}^{0}$ which is equal to $\Omega$ in the region $\mathcal{R}$, and falls off rapidly in the region of moduli space where we pull the two monopoles in the cluster away from each other at a distance $>>K$.

Let $h$ denote the action of the first $Z_{2}$ transformation on $\mathcal{M}_{2}^{0}$. As explained in appendix B, this corresponds to moving one of the monopoles in the cluster by a distance $\pi R_{(3)}$ along the positive 3-axis, and the other monopole by a distance $-\pi R_{(3)}$ along the negative 3 -axis. Let $h \circ \widehat{\Omega}$ denote the action of the diffeomorphism $h$ on the approximate harmonic form $\widehat{\Omega}$. Since the diffeomorphism generated by $h$ is a symmetry of the metric, $h \circ \widehat{\Omega}$ is also an approximate harmonic form on $\mathcal{M}_{2}^{0}$. Furthermore, since $\widehat{\Omega}$ has support mostly in the region of $\mathcal{M}_{2}^{0}$ where the monopoles cluster around the points $2 \pi n R_{(3)}$, and (as follows from the definition of $h$ ) $h \circ \widehat{\Omega}$ has support mostly in the region of $\mathcal{M}_{2}^{0}$ where the monopoles cluster around the 
points $(2 n+1) \pi R_{(3)}$, there is no significant overlap between the forms $\widehat{\Omega}$ and $h \circ \widehat{\Omega}$. It then follows that

$$
\omega^{(k)} \equiv \sum_{l=0}^{1} e^{i \pi k l} h^{l} \circ \widehat{\Omega}
$$

are also approximate harmonic forms on $\mathcal{M}_{2}^{0}$ with the property that $\omega^{(k)}$ picks up a phase of $\exp (i \pi k)$ under the action of the first $Z_{2}$ group of transformations generated by $h$, and a phase of -1 under the second $Z_{2}$ group of transformations associated with the $\mathrm{U}(1)$ phase. The construction given in eq.(4.4) gives two independent $\omega^{(k)}$, which can be taken to be $\omega^{(0)}$ and $\omega^{(1)}$.

Normally one would expect that the above approximate harmonic forms on $\mathcal{M}_{2}^{0}$ can be suitably modified so as to give exact eigenforms $\bar{\omega}^{(k)}$ of the Laplacian with a small, but not necessarily zero, eigenvalue $\lambda^{(k)}$. Physically this effect may be understood by regarding the system as a one dimensional periodic array of two monopole bound states (with finite binding energy). We expect the bound state energy to shift slightly due to the influence of the other monopoles and the periodicity requirement, but do not expect the state to disappear completely. We shall now argue that in the present case $\lambda^{(k)}$ vanishes exactly. This is proved by noting that if $\lambda^{(k)} \neq 0$, then either $d$ or $\delta \equiv * d *$ (or both) does not annihilate $\bar{\omega}^{(k)}$. Let us assume that $d \bar{\omega}^{(k)} \neq 0$; the case where $\delta \bar{\omega}^{(k)} \neq 0$ can be dealt with in an identical manner. We can then define a normalized differential form $\widetilde{\omega}^{(k)} \equiv d \bar{\omega}^{(k)} / \sqrt{\lambda^{(k)}}$ which is also an eigenform of the laplacian with eigenvalue $\lambda^{(k)}$. Since for $R_{(3)} \rightarrow \infty, \lambda^{(k)} \rightarrow 0$, in this limit $\widetilde{\omega}^{(k)}$ corresponds to a harmonic form on $\mathcal{M}_{2}^{0}$, and its restriction to $\mathcal{R}$ would correspond to a new harmonic form on the two monopole moduli space $M_{2}^{0 \star}$ (Note that in this limit $\widetilde{\omega}^{(k)}$ ceases to be an exact form, since $d \bar{\omega}^{(k)} \rightarrow 0$.) But we know from the analysis of the two monopole moduli space that such harmonic forms do not exist [10]. This shows that our initial assumption must be wrong, i.e. $\lambda^{(k)}$ must vanish.

$\star$ We are implicitly assuming that $\widetilde{\omega}^{(k)}$ will also have its support mostly inside $\mathcal{R}$ and $h(\mathcal{R})$. This is physically plausible, since bound states of monopoles with finite binding energy are expected to have support only in regions where the monopoles are close to each other. 
This argument shows the existence of the relevant harmonic forms $\bar{\omega}^{(k)}$ on $\mathcal{M}_{2}^{0}$ for large $R_{(3)}$ and hence the existence of the relevant supersymmetric state in the two soliton sector. It is probably clear to the reader that the argument presented here is simply a variation of the argument [13] that once $\lambda^{(k)}$ is nonzero, the number of states increase, and there is no convenient way of getting rid of these extra states in the $R_{(3)} \rightarrow \infty$ limit. We can now use the same continuity argument as in ref.[13] to argue that these states, and hence these harmonic forms must exist also for finite values of $R_{(3)}$. Alternatively, one could argue that the number of square integrable harmonic forms on a manifold is a topological property of the manifold, and is not expected to change under smooth deformations of the manifold.

As in the case of four dimensional theories [6] [7], we can also give a plausibility argument for the existence of these bound states based on triangle inequality. Since the Bogomol'nyi formula gives infinite mass of all the states, this is not a useful starting point. Instead, we shall base our calculation on the direct analysis of the force between far away solitons. For this, let us consider two arrays, one carrying $r_{1}$ units of magnetic charge and $p_{1}$ units of electric charge per unit period in the third direction, and the other carrying $r_{2}$ units of magnetic charge and $p_{2}$ units of electric charge per unit period in the third direction. Using the results of ref.[36] giving the asymptotic Higgs and gauge fields around a dyon, one can show that the attractive force (proportional to the inverse separation) due the exchange of massless Higgs between the two arrays is proportional to $\sqrt{\left(p_{1}\right)^{2}+\left(r_{1}\right)^{2}} \sqrt{\left(p_{2}\right)^{2}+\left(r_{2}\right)^{2}}$, whereas the repulsive electric and magnetic force between the two arrays is proportional to $\left(p_{1} p_{2}+r_{1} r_{2}\right){ }^{\dagger}$ These two forces balance each other only when the two vectors $\left(\begin{array}{l}p_{1} \\ r_{1}\end{array}\right)$ and $\left(\begin{array}{l}p_{2} \\ r_{2}\end{array}\right)$ are proportional to each other. Otherwise the attractive force due to the higgs exchange is always larger then the repulsive electric and magnetic force.

\footnotetext{
$\dagger$ For simplicity, we have assumed that the coupling constant is unity, but this is not a necessary assumption.
} 
Let us now consider a state carrying $p$ units of magnetic charge and $r$ units of electric charge per unit period in the three direction. If $p$ and $r$ are relatively

prime, it is clear that the vector $\left(\begin{array}{l}p \\ r\end{array}\right)$ cannot be written as a sum of two other vectors with integer entries which are proportional to each other. In other words, if we try to regard the array carrying the above magnetic and electric charges as a combination of two different arrays, then for large separation, these two arrays experience a net attractive force. It is then plausible that there are bound states of these two arrays, and that there exists specific bound states which attain the lowest possible energy, i.e. saturate the Bogomol'nyi bound. These are precisely the states that are needed for establishing duality invariance of the spectrum.

\section{Fundamental Strings and Stringy Cosmic Strings}

There are two kinds of different string like solutions that have been constructed in four dimensional string theory. The first kind, known as fundamental string, describes the classical field configuration around a fundamental string and was constructed in ref.[15]. The second kind, which was called 'stringy cosmic string', and was constructed in ref.[25][37], represents a solution where one of the 6 compact directions decompactify at the core of the string. Each of these two solutions may be regarded as a solution in the three dimensional string theory by taking the direction, along which the string extends, to be compact. In this section we shall show that these two different classes of three dimensional solutions are, in fact, related by an $\mathrm{O}(8,24 ; \mathrm{Z})$ transformation. Unlike in the previous section, our discussion in this section will be based on the study of the classical solutions themselves, but we expect that this can be elevated to the quantum level once we properly quantize the collective coordinate excitations of the corresponding solutions.

We begin with a discussion of the fundamental string solution. In the notation of this paper, the field configuration associated with a fundamental string solution, 
winding once in the three direction and without carrying any other charges, is given by,

$$
\begin{aligned}
& \mathcal{M}=\left(\begin{array}{ccccccc}
\frac{|\lambda|^{2}}{\lambda_{2}} & 0 & 0 & 0 & 0 & \frac{\lambda_{1}}{\lambda_{2}} & 0 \\
0 & I_{6} & 0 & 0 & 0 & 0 & 0 \\
0 & 0 & \frac{1}{\lambda_{2}} & 0 & 0 & 0 & -\frac{\lambda_{1}}{\lambda_{2}} \\
0 & 0 & 0 & I_{6} & 0 & 0 & 0 \\
0 & 0 & 0 & 0 & I_{16} & 0 & 0 \\
\frac{\lambda_{1}}{\lambda_{2}} & 0 & 0 & 0 & 0 & \frac{1}{\lambda_{2}} & 0 \\
0 & 0 & -\frac{\lambda_{1}}{\lambda_{2}} & 0 & 0 & 0 & \frac{|\lambda|^{2}}{\lambda_{2}}
\end{array}\right) \\
& g_{\mu \nu} d x^{\mu} d x^{\nu}=-d t^{2}+\lambda_{2}|d z|^{2},
\end{aligned}
$$

where

$$
\lambda \equiv \lambda_{1}+i \lambda_{2}=\frac{i}{2 \pi} \ln \frac{A}{z} .
$$

$z=x^{1}+i x^{2}$ denotes the complex coordinate labelling the two dimensional space, and $A$ is an arbitrary complex number. Note that this solution is ill defined for large $|z|$, since $\lambda_{2}$ becomes negative, forcing $\Phi$ to be complex. Thus the solution given above should be regarded only as an approximate solution that gets modified at large distances when $\lambda_{2}$ becomes of order 1 and the theory enters the strong coupling regime. We note in passing that the $\mathrm{SL}(2, \mathrm{Z})$ transform of these solutions with the $\mathrm{O}(8,24 ; \mathrm{Z})$ matrices $\Omega$ given in eq.(2.26) will correspond to magnetically charged strings discussed in ref.[38], winding along the $x^{3}$ direction.

Let us now consider the $\mathrm{O}(8,24 ; \mathrm{Z})$ transformation $\omega$ that exchanges the 2 nd 
and 31st rows and columns, and also the 9th and 32nd rows and columns:

$$
\omega=\left(\begin{array}{cccccccc}
1 & 0 & 0 & 0 & 0 & 0 & 0 & 0 \\
0 & 0 & 0 & 0 & 0 & 0 & 1 & 0 \\
0 & 0 & I_{5} & 0 & 0 & 0 & 0 & 0 \\
0 & 0 & 0 & 1 & 0 & 0 & 0 & 0 \\
0 & 0 & 0 & 0 & 0 & 0 & 0 & 1 \\
0 & 0 & 0 & 0 & 0 & I_{21} & 0 & 0 \\
0 & 1 & 0 & 0 & 0 & 0 & 0 & 0 \\
0 & 0 & 0 & 0 & 1 & 0 & 0 & 0
\end{array}\right) .
$$

This transforms the solution (5.1) to

$$
\mathcal{M}=\left(\begin{array}{cccccc}
\frac{|\lambda|^{2}}{\lambda_{2}} & \frac{\lambda_{1}}{\lambda_{2}} & 0 & 0 & 0 & 0 \\
\frac{\lambda_{1}}{\lambda_{2}} & \frac{1}{\lambda_{2}} & 0 & 0 & 0 & 0 \\
0 & 0 & I_{5} & 0 & 0 & 0 \\
0 & 0 & 0 & \frac{1}{\lambda_{2}} & -\frac{\lambda_{1}}{\lambda_{2}} & 0 \\
0 & 0 & 0 & -\frac{\lambda_{1}}{\lambda_{2}} & \frac{|\lambda|^{2}}{\lambda_{2}} & 0 \\
0 & 0 & 0 & 0 & 0 & I_{23}
\end{array}\right)
$$

with $g_{\mu \nu}$ and $\lambda$ still given by eqs.(5.2) and (5.3) respectively. Using the relations (2.3), (2.4), (2.12) and (2.14) between the matrix $\mathcal{M}$ and the ordinary metric and antisymmetric tensor fields in ten dimension, it can easily be checked that this corresponds to a ten dimensional metric of the form:

$$
\left(\begin{array}{ll}
G_{33}^{(10)} & G_{34}^{(10)} \\
G_{43}^{(10)} & G_{44}^{(10)}
\end{array}\right)=\frac{1}{\lambda_{2}}\left(\begin{array}{cc}
1 & -\lambda_{1} \\
-\lambda_{1} & |\lambda|^{2}
\end{array}\right) .
$$

This soluion coincides with the 'stringy cosmic string solution' constructed in ref.[25] near the 'decompactification point' (small $|z|$ ). At $z=0$ the direction 4 decompactifies, since the metric component $G_{44}^{(10)}$ blows up. Note the amusing fact that in order to identify this solution to the four dimensional solution of 
ref.[25], we need to identify the coordinates $\left(x^{0}, x^{1}, x^{2}\right)$, and any one of the coordinates $\left(x^{5}, \ldots x^{9}\right)$ (let us choose $x^{5}$ for definiteness) as the coordinates of the four dimensional theory, with the string extending in the $x^{5}$ direction. But the coordinate $x^{3}$, which was the direction in which the original fundamental string was pointing, now must be regarded as a compact direction to start with and cannot be taken to be the direction in which the cosmic string extends.

The solution given in eq.(5.5), or equivalently eq.(5.6), suffers from the same kind of problems at large distances as the solution (5.1), since eigenvalues of the metric become negative. An elegant solution to this problem was provided in ref.[25]. Let $j(\lambda)$ be the SL(2,Z) invariant function of the variable $\lambda$, satisfying

$$
\begin{aligned}
j\left(\frac{p \lambda+q}{r \lambda+s}\right) & =j(\lambda) \quad \text { for } \quad p s-r q=1, \quad p, q, r, s \in Z, \\
j(\lambda) & \simeq e^{-2 \pi i \lambda} \text { for large } \lambda_{2},
\end{aligned}
$$

and that $j(\lambda)$ has no other pole anywhere else in the upper half $\lambda$ plane. Let us now consider a field configuration given by eq.(5.5), with $\lambda(z)$ given by,

$$
j(\lambda(z))=\frac{A}{z}+B
$$

Then, for $|z|<<|A|$, we get back eq.(5.3). This shows that for small $|z|$ the new solution agrees with the original one. However, since $\lambda(z)$ is obtained as a solution to eq.(5.8), $\lambda_{2}$ is guaranteed to be positive for all values of $z$. Furthermore, as $|z| \rightarrow \infty, \lambda$ approaches a constant value, depending on the value of $B$. As a result, this solution is well behaved asymptotically.

This suggests that we should modify the fundamental string solution at large distance in a similar way, so that it has good asymptotic behaviour. In particular, we can now take the fundamental string solution to be given by eq.(5.1), with $\lambda$ given by eq.(5.8) instead of eq.(5.3). This agrees with the original solution for small $|z|$, but differs from it for large $|z|$, and is free from the problem of the original 
solution. For $\lambda$ given as a solution of eq.(5.8), $\mathcal{M}$ is not a single valued function of $z$. In particular, there are closed curves in the $z$ plane, not necessarily enclosing the string, but the points where $\lambda=i$ or $\exp ( \pm 2 \pi i / 3)$, with the property that $\lambda$ transforms by an $\mathrm{SL}(2, \mathrm{Z})$ transformation of the form $\lambda \rightarrow(p \lambda+q) /(r \lambda+s)$ as we move around these closed curves. This induces a transformation on $\mathcal{M}$ of the form:

$$
\mathcal{M} \rightarrow \Omega \mathcal{M} \Omega^{T}
$$

where,

$$
\Omega=\left(\begin{array}{ccccccc}
p & 0 & 0 & 0 & 0 & q & 0 \\
0 & I_{6} & 0 & 0 & 0 & 0 & 0 \\
0 & 0 & s & 0 & 0 & 0 & -r \\
0 & 0 & 0 & I_{6} & 0 & 0 & 0 \\
0 & 0 & 0 & 0 & I_{16} & 0 & 0 \\
r & 0 & 0 & 0 & 0 & s & 0 \\
0 & 0 & -q & 0 & 0 & 0 & p
\end{array}\right) .
$$

Since $\Omega$ is an $\mathrm{O}(8,24 ; \mathrm{Z})$ transformation, we see that the solution obtained this way is well defined if $\mathrm{O}(8,24 ; \mathrm{Z})$ is a gauge symmetry of the theory, so that we can identify field configurations related by this transformation.

Although the solution constructed this way is well defined at this level, it was argued in ref.[25] that as a result of the presence of the orbifold points in the map from the $z$ plane to the $\lambda$ plane, corresponding to the points $\lambda=i$ or $\lambda=\exp ( \pm 2 \pi i / 3)$, stringy modification of these solutions, which preserve partial invariance of these solutions under supersymmetry transformations, may not be possible. It was also shown in ref.[25] that there exists 12 string configurations which do not suffer from this difficulty. This might mean that single elementary string states do not represent well defined states, but 12 of them together do.

$\star$ I wish to thank A. Shapere for a discussion on this point.

$\dagger$ The argument in ref.[25] was given not for the fundamental string solutions, but for the cosmic string solutions. However, since these two solutions are related by an $\mathrm{O}(8,24 ; \mathrm{Z})$ transformation, the same argument must apply to this case as well. 
We end this section by mentioning that the mechanism of replacing eq.(5.3) by eq.(5.8), that makes the solution well behaved asymptotically, might also be responsible for the removal of infra-red divergences alluded to in sects. 3 and 4 from other string states as well. As stated there, these divergences are due to the long range electric and magnetic fields around the elementary string and soliton states. Equivalently we can attribute these divergences to the fact that the field $\mathcal{M}$ does not approach a constant value asymptotically; this can be seen from eqs.(3.2) and (3.6). This situation is very similar to that in the solution (5.3), where $\lambda$ does not approach a constant value asymptotically. In particular, $\lambda \rightarrow \lambda+1$ under

$z \rightarrow e^{2 \pi i} z$. Thus it is conceivable that using the identification of field configurations under the $\mathrm{O}(8,24 ; \mathrm{Z})$ symmetry, the field configurations around the elementary string excitations and solitons can be appropriately modified (as in eq.(5.8)) so that $\mathcal{M}$ approaches some constant value $\mathcal{M}^{(0)}$ asymptotically. Such solutions will be free from the infra-red divergence problems discussed in the previous section.

\section{APPENDIX A}

\section{Asymptotic Behaviour of the Periodic Array of BPS Monopole Solutions}

In this appendix we shall discuss the asymptotic field configurations for a periodic array of BPS monopole solutions. We first consider a configuration of $N$ well separated monopole solutions in four dimensions, and work in a gauge where asymptotically the Higgs field is directed along a fixed direction (say the 3 direction) in the isospin space everywhere except along a Dirac string singularity. The asymptotic magnetic field is given by

$$
\vec{B} \simeq \sum_{I=1}^{N} \frac{\vec{r}-\vec{r}_{(0) I}}{\left|\vec{r}-\vec{r}_{(0) I}\right|^{3}} \frac{\sigma_{3}}{2},
$$

where $\vec{r}_{(0) I}$ is the location of the Ith monopole. The Bogomol'nyi equation

$$
D_{i} \phi= \pm B_{i}
$$

for the Higgs field $\phi$ then determines the asymptotic value of the Higgs field upto 
an additive constant. Choosing the + sign in eq.(A.2) we get,

$$
\phi \simeq\left[C-\sum_{I=1}^{N} \frac{1}{\left|\vec{r}-\vec{r}_{(0) I}\right|}\right] \frac{\sigma_{3}}{2}
$$

Here $C$ is a constant which sets the scale of breaking of the $\mathrm{SU}(2)$ symmetry.

From this we can construct the asymptotic form for the Higgs field for a periodic array of BPS monopoles along the three direction provided the period is large compared to the scale of $\mathrm{SU}(2)$ breaking. It is given by,

$$
\phi \simeq\left[C-\sum_{k=-\infty}^{\infty} \frac{1}{\sqrt{\left(x^{1}-x_{(0)}^{1}\right)^{2}+\left(x^{2}-x_{(0)}^{2}\right)^{2}+\left(x^{3}-x_{(0)}^{3}+2 \pi k R_{(3)}\right)^{2}}}\right] \frac{\sigma_{3}}{2} .
$$

The sum over $k$ in this expression has a logarithmic divergence, but the divergene contribution is independent of the coordinates $\left(x^{1}, x^{2}, x^{3}\right)$ and can be removed by appropriately adjusting $C$. The asymptotic values of the gauge field strengths are given by eq.(A.2), and are finite. This way we can construct a soliton solution in the three dimensional string theory from periodic array of BPS monopoles in four dimensions. The solution, however, suffers from the usual problem of logarithmic growth of the scalar field $\phi$ at large distance, and the associated problem of infinite energy. In particular, if we define,

$$
\rho=\sqrt{\left(x^{1}-x_{(0)}^{1}\right)^{2}+\left(x^{2}-x_{(0)}^{2}\right)^{2}},
$$

then for large $\rho$,

$$
\phi \simeq \frac{1}{2 \pi R_{(3)}}(\ln \rho) \sigma_{3}
$$

The $N$-soliton solution in three dimensions can be constructed in an identical manner from $N$ different periodic arrays of BPS monopoles, and has the following 
asymptotic form of the Higgs field when the arrays are well separated from each other:

$$
\phi \simeq\left[C-\sum_{I=1}^{N} \sum_{k=-\infty}^{\infty} \frac{1}{\sqrt{\left(x^{1}-x_{(0) I}^{1}\right)^{2}+\left(x^{2}-x_{(0) I}^{2}\right)^{2}+\left(x^{3}-x_{(0) I}^{3}+2 \pi k R_{(3)}\right)^{2}}}\right] \frac{\sigma_{3}}{2} .
$$

Finally, we would like to point out that although these solutions are not independent of the coordinate $x^{3}$, and hence are not vortex solutions in the conventional sense, the dependence of these solutions on $x^{3}$ decreases exponentially as we move away from the core. To see this, let us consider a single array of monopoles. In this case, the asymptotic Higgs field is given by eq.(A.4), and hence,

$$
\begin{aligned}
\frac{\partial \phi}{\partial x^{3}} & \simeq \sum_{k=-\infty}^{\infty} \frac{\left(x^{3}-x_{(0)}^{3}+2 \pi k R_{(3)}\right)}{\left(\rho^{2}+\left(x^{3}-x_{(0)}^{3}+2 \pi k R_{(3)}\right)^{2}\right)^{3 / 2}} \cdot \frac{\sigma_{3}}{2} \\
& =\oint \frac{d w}{2 i} \cot (\pi w) \frac{\left(x^{3}-x_{(0)}^{3}+2 \pi w R_{(3)}\right)}{\left(\rho^{2}+\left(x^{3}-x_{(0)}^{3}+2 \pi w R_{(3)}\right)^{2}\right)^{3 / 2}} \cdot \frac{\sigma_{3}}{2},
\end{aligned}
$$

where the contour of integration over $w$ encloses the real axis. We can now express this as the sum of two contours, $C_{1}$ and $C_{2}$, given by,

$$
\begin{array}{lll}
w=y-\frac{1}{2 \pi R_{(3)}}\left\{\left(x^{3}-x_{(0)}^{3}\right)-i(\rho-\epsilon)\right\} & \text { on } C_{1}, & \\
w=-y-\frac{1}{2 \pi R_{(3)}}\left\{\left(x^{3}-x_{(0)}^{3}\right)+i(\rho-\epsilon)\right\} & \text { on } C_{2}, & -\infty<y<\infty, \quad y \text { real, }
\end{array}
$$

where $\epsilon$ is a small but fixed number. Eq.(A.8) may then be rewritten as

$$
\begin{aligned}
\frac{\partial \phi}{\partial x^{3}} \simeq & \int_{-\infty}^{\infty} \frac{d y}{2 i} \frac{2 \pi R_{(3)} y+i(\rho-\epsilon)}{\left(\left\{2 \pi R_{(3)} y+i(2 \rho-\epsilon)\right\}\left\{2 \pi R_{(3)} y-i \epsilon\right\}\right)^{3 / 2}} \\
& \times\left[\cot \left\{\frac{i(\rho-\epsilon)-\left(x^{3}-x_{(0)}^{3}\right)}{2 R_{(3)}}+\pi y\right\}-\cot \left\{\frac{i(\rho-\epsilon)+\left(x^{3}-x_{(0)}^{3}\right)}{2 R_{(3)}}+\pi y\right\}\right] \frac{\sigma_{3}}{2},
\end{aligned}
$$

where the factor $\left(\left\{2 \pi R_{(3)} y+i(2 \rho-\epsilon)\right\}\left\{2 \pi R_{(3)} y-i \epsilon\right\}\right)^{3 / 2}$ in the denominator is to be taken to be positive for $y=0$. This defines this term on the real $y$ axis 
completely since there is no branch point on this axis. The term inside the square bracket is of order $e^{-(\rho-\epsilon) / R_{(3)}}$ for large $\rho$. The rest of the integrand is finite and falls off as $y^{-2}$ for large $|y|$. This shows that $\left(\partial \phi / \partial x^{3}\right)$ falls off exponentially as we move away from the soliton core.

We end with the note that our analysis so far does not show that the solutions representing periodic arrays of BPS monopoles, constructed this way, are nonsingular everywhere in space. As we shall see in appendix B, these solutions give rise to well defined scattering functions, which might be an indication that the solutions are indeed finite everywhere in space. In particular, if the solution is singular at some point, then we might expect the scattering function associated with a line passing through that point to be ill defined. Even if it turns out that the solutions have singularity at some point(s) in space, we might hope that stringy corrections will remove the singularity, or that string propagation in the background provided by these solutions is well behaved, even if the solutions themselves are singular.

\section{APPENDIX B}

\section{Moduli Space of Periodic Arrays of BPS Multi-monopole Solutions}

In this appendix we study the moduli space of periodic array of BPS multimonopole solutions in $(3+1)$ dimensions. We start with the observation [39] that moduli space of $N$ monopole solutions is identical to the space of rational functions $S(v)=P(v) / Q(v)$, where $P(v)$ is a polynomial in $v$ of degree $\leq N-1, Q(v)$ is a polynomial in $v$ of degree $N$, and the zeroes of $Q(v)$ are distinct from the zeroes of $P(v)$. For $N$ well separated monopoles the zeroes of $Q(v)$ are all distinct, and $S(v)$ has the form:

$$
S(v)=\sum_{I=1}^{N} \frac{\lambda_{I}}{v-v_{I}}
$$

$S(v)$ has the interpretation of being the scattering function associated with the the differential operator $\partial_{t}+i A_{t}-\phi$ on a line parallel to the $x^{2}$ axis, where $t$ denotes 
length measured along the line, and $v=x^{3}+i x^{1}$ labels the point of intersection of the line with the $1-3$ plane. Thus, a periodic array of monopoles along the 3 axis with period $2 \pi R_{(3)}$ will be described by a scattering function of the form:

$$
S(u)=\sum_{n=-\infty}^{\infty} \frac{\lambda}{u-u_{0}-2 \pi n}=\frac{\lambda}{2} \cot \left[\frac{1}{2}\left(u-u_{0}\right)\right]
$$

where $u \equiv\left(x^{3}+i x^{1}\right) / R_{(3)}$. It is also straightforward to write down the scattering function $S(u)$ for $N$ well separated arrays of BPS monopoles. This is given by,

$$
S(u)=\frac{1}{2} \sum_{I=1}^{N} \lambda_{I} \cot \left[\frac{1}{2}\left(u-u_{I}\right)\right], \quad u_{I} \neq u_{J} \text { for } I \neq J
$$

This function is parametrized by $2 N$ complex variables $\lambda_{I}$ and $u_{I}$.

The function $S(u)$ given in eq.(B.3) is characterized by the fact that it is periodic in $\operatorname{Re}(u)$ with period $2 \pi$, it has $N$ distinct poles in the interval $0 \leq R e(u)<$ $2 \pi$, and that $\lim _{u \rightarrow i \infty} S(u)=-\lim _{u \rightarrow-i \infty} S(u)$, but neither of these limits vanish. A more well behaved scattering function, one that vanishes for $\operatorname{Im}(u) \rightarrow \pm \infty$, may be obtained if we consider quasi-periodic arrays of monopoles which pick up a $\mathrm{U}(1)$ phase $e^{2 \pi i \alpha}$ under translation by one period. ${ }^{\dagger}$ A single array of this kind is described by a scattering function

$$
S(u)=\sum_{n=-\infty}^{\infty} \frac{\lambda e^{2 \pi i \alpha n}}{u-u_{0}-2 \pi n}=\frac{\lambda}{2} \frac{e^{i\left(\alpha-\frac{1}{2}\right)\left(u-u_{0}\right)}}{\sin \left[\frac{1}{2}\left(u-u_{0}\right)\right]}, \quad \text { for } 0<\alpha<1
$$

(Up to an overall normalization factor, this result may also be derived by starting from the configuration of cyclically symmetric monopoles given in ref.[40] and then

\footnotetext{
$\star$ Instead of choosing the lines to be parallel to the $x^{2}$ axis, we could have chosen any other set of parallel lines. For a detailed discussion of this see Ref.[39].

$\dagger$ This corresponds to introducing a Wilson line along the 3 direction [8].
} 
taking the limit where the radius of the circle becomes large.) A configuration of multiple well separated arrays is described by the scattering function:

$$
S(u)=\frac{1}{2} \sum_{I=1}^{N} \lambda_{I} \frac{e^{i\left(\alpha-\frac{1}{2}\right)\left(u-u_{I}\right)}}{\sin \left[\frac{1}{2}\left(u-u_{I}\right)\right]}, \quad \text { for } 0<\alpha<1
$$

Note that $S(u)$ vanishes for $\operatorname{Im}(u) \rightarrow \pm \infty$. This fall-off may be attributed to the cancellation between the contributions from different monopoles in the array due to their phase difference. In analogy with the BPS monopole solutions in four dimensions, we now speculate that for the most general configuration of $N$ quasiperiodic arrays (not necessarily well-separated) of this kind, the scattering function $S(u)$ satisfies the following criteria:

1. $S(u+2 \pi)=e^{2 \pi i \alpha} S(u)$.

2. $\lim _{u \rightarrow \pm i \infty} S(u)=0$.

3. $S(u)$ has $N$ (not necessarily distinct) poles in the interval $0 \leq R e(u)<2 \pi$.

A general expression for such a function is given by

$$
S(u)=\frac{e^{i(\alpha+N / 2) u} \sum_{J=1}^{N} a_{J} e^{-i J u}}{\prod_{I=1}^{N} \sin \left[\frac{1}{2}\left(u-u_{I}\right)\right]},
$$

with the constraint

$$
\prod_{I=1}^{N} \sum_{J=1}^{N} a_{J} e^{-i J u_{I}} \neq 0
$$

Eq.(B.7) guarantees that the poles in $S(u)$ coming from the zeroes in the denominator are not cancelled by the zeroes of the numerator. We shall denote by $\mathcal{M}_{N}$ the $4 N$ real dimensional space parametrized by $a_{I} \in C$ and $u_{I} \in S^{1} \times R$, satisfying (B.7).

There is a natural action of the group $R^{2} \times S^{1} \times S^{1}$ on the function $S(u)$ and hence on the space $\mathcal{M}_{N}$. This group action is identical to that for $N$ monopole solutions in four dimensions, with the only difference that the translation in the 
third direction is now represented by a compact coordinate. If $\left(X^{1}, X^{2}, X^{3}, \Theta\right)$ denote the parameters of the group, with $X^{3}$ and $\Theta$ periodic parameters with period $2 \pi$, then under the action of this group,

$$
S(u) \rightarrow e^{X^{2}+i \Theta} S\left(u-X^{3}-i X^{1}\right)
$$

Physically, $X^{1}, X^{2}$ and $X^{3}$ denote translations in the 1,2 and 3 directions respectively, while $\Theta$ represents a change of the $U(1)$ phase. Using these symmetry transformations, we can locally 'gauge fix' the coordinates $a_{I}, u_{I}$ of the full moduli space $\mathcal{M}_{N}$ to $\widetilde{a}_{I}, \widetilde{u}_{I}$, satisfying,

$$
\sum_{I=1}^{N} \widetilde{u}_{I}=0
$$

and,

$$
\prod_{I=1}^{N} \sum_{J=1}^{N} \widetilde{a}_{J} e^{-i J \widetilde{u}_{I}}=1
$$

We shall denote by $\mathcal{M}_{N}^{0}$ the $4(N-1)$ dimensional space spanned by the coordinates $\tilde{a}_{J} \in C$ and $\tilde{u}_{I} \in S^{1} \times R$, satisfying eqs.(B.9), (B.10). Then locally the full moduli space $\mathcal{M}_{N}$ parametrized by the coordinates $a_{I}$ and $u_{I}$ subject to the constraint (B.7) can be described the coordinates $\left(X^{1}, X^{2}, X^{3}, \Theta\right)$ of $R^{2} \times S^{1} \times S^{1}$ and those of $\mathcal{M}_{N}^{0}$ through the relations:

$$
a_{J}=\widetilde{a}_{J} e^{X^{2}+i \Theta} e^{i J\left(X^{3}+i X^{1}\right)}
$$

and,

$$
u_{I}=\widetilde{u}_{I}+X^{3}+i X^{1}
$$

Thus locally the full moduli space $\mathcal{M}_{N}$ has the structure of $R^{2} \times S^{1} \times S^{1} \times \mathcal{M}_{N}^{0}$. Globally, however, we need a $Z_{N} \times Z_{N}$ identification of the points in this space to 
recover the correct moduli space. This is seen by noting that the following $Z_{N} \times Z_{N}$ transformations in $R^{2} \times S^{1} \times S^{1} \times \mathcal{M}_{N}^{0}$ leave the original parameters $a_{I}$ and $u_{I}$ unchanged (up to shift of $u_{I}$ by multiples of $2 \pi$ ):

$$
\begin{aligned}
& \widetilde{u}_{I} \rightarrow \widetilde{u}_{I}+\frac{2 \pi}{N} \quad \text { for } \quad 1 \leq I \leq N-1, \quad \widetilde{u}_{N} \rightarrow \widetilde{u}_{N}+\frac{2 \pi}{N}-2 \pi, \\
& \widetilde{a}_{J} \rightarrow \widetilde{a}_{J} e^{\frac{2 \pi i J}{N}}, \quad X^{3} \rightarrow X^{3}-\frac{2 \pi}{N},
\end{aligned}
$$

and,

$$
\widetilde{a}_{J} \rightarrow \widetilde{a}_{J} e^{\frac{2 \pi i}{N}}, \quad \Theta \rightarrow \Theta-\frac{2 \pi}{N}
$$

This establishes the structure (4.3) of the moduli space of multiple array of BPS monopole solutions. For well separated arrays, the transformation (B.13) corresponds to translating the $N$ th array by a distance $2 \pi$ along the negative 3 -axis relative to the other arrays, accompanied by an overall shift of all the arrays by a distance $2 \pi / N$ along the positive 3 -axis.

\section{APPENDIX C}

\section{Metric on $\mathcal{M}_{N}$}

We shall begin by discussing a subtlety involving the overall $\mathrm{U}(1)$ phase $\Theta$. A change of $\Theta$ by $\delta \Theta$ corresponds to an infinitesimal gauge transformation of the solution with matrix valued parameter $\Lambda\left(x^{1}, x^{2}, x^{3}\right) \equiv \sum_{a=1}^{3} \Lambda^{a} \sigma^{a} / 2$ which approaches the value $\delta \Theta \sigma_{3} / 2$ far away from the soliton core. In order to see what kind of deformation of the solution it corresponds to, we run into a problem. In we demand as in ref.[39] that the deformation generated by the gauge transformation parameter $\Lambda$ is orthogonal to all the pure gauge deformations ${ }^{\star}$ then $\Lambda$ must be

\footnotetext{
$\star$ In this case orthogonality is only in a formal sense, since the proof of orthogonality given in ref.[39] involves an integration by parts, and the relevant boundary terms do not vanish in this case.
} 
proportional to the background Higgs field $\phi[39]$ :

$$
\Lambda\left(x^{1}, x^{2}, x^{3}\right)=\delta \widehat{\Theta} \phi\left(x^{1}, x^{2}, x^{3}\right),
$$

where $\delta \widehat{\Theta}$ is a free parameter. Eqs.(A.7) and (C.1) shows that asymptotically $\Lambda \simeq$ $\left(N / 2 \pi R_{(3)}\right) \delta \widehat{\Theta}(\ln \rho) \sigma_{3}$. Comparing this with the asymptotic form of $\Lambda$ generated by the deformation of the parameter $\Theta$, we see that the deformation induced by $\delta \widehat{\Theta}$ is not compatible with the deformation induced by $\delta \Theta$. Furthermore, as we shall see later, a deformation induced by $\Lambda$ given in eq.(C.1)(as well as the deformations induced by the parameters $X^{1}$ and $X^{2}$ ) are not normalizable. All these difficulties may be avoided by introducing an explicit infra-red cut-off at some length scale $L$. In this case all the deformations will be normalizable, and the parameter $\Lambda$ given by eq.(C.1) approaches the value $\left(N / 2 \pi R_{(3)}\right) \delta \widehat{\Theta}(\ln L) \sigma_{3}$ asymptotically. Thus now $\widehat{\Theta}$ and $\Theta$ can be related as $\Theta=N \widehat{\Theta} \ln L / \pi R_{(3)}$.

Such a regularization may be provided, for example, by keeping the extent of the array in the third direction to be large but finite, instead of being strictly periodic and infinite. If the array stops after $L$ repetitions, then locally $\mathcal{M}_{N}$ can be approximately identified to a $4 N$ dimensional subspace of the $4 N L$ dimensional moduli space $M_{N L}$ of $N L$ monopoles in $3+1$ dimensions. Equivalently, one could consider a $Z_{L}$ symmetric cyclic arrangement [40] of $N L$ monopoles around a circle of radius $L R_{(3)}$ in the $x^{1}-x^{3}$ plane, each monopole being a finite distance away from the circle. In the $L \rightarrow \infty$ limit this represents $N$ periodic arrays of monopoles with periodicity $2 \pi R_{(3)}$.

Using arguments similar to those in ref.[39] one can show that the vector fields $\partial / \partial X^{1}, \partial / \partial X^{2}, \partial / \partial X^{3}$ and $\partial / \partial \widehat{\Theta}$ are covariantly constant in the full moduli space. As a result, the metric on the full moduli space $\mathcal{M}_{N}$ factorizes into a direct sum of a constant metric on $R^{2} \times S^{1} \times S^{1}$ and a metric on $\mathcal{M}_{N}^{0}$. We shall now show that although the constant metric on $R^{2} \times S^{1} \times S^{1}$ depends on the infra-red regulator $L$, the metric on $\mathcal{M}_{N}^{0}$ is in fact independent of $L$ and hence the notion of the existence of harmonic forms on $\mathcal{M}_{N}^{0}$ is well defined. We first use eq.(A.7) to 
express the asymptotic value of the Higgs field as

$$
\phi=\left\{\widetilde{C}+\frac{N}{\pi R_{(3)}} \ln |\vec{\rho}-\vec{R}|+O\left(\frac{1}{\rho^{2}}\right)\right\} \frac{\sigma_{3}}{2}+\sum_{i=1}^{2} \sigma_{i} f_{i}(\vec{\rho}),
$$

where,

$$
\begin{gathered}
\vec{R} \equiv\left(R_{(3)} X^{1}, R_{(3)} X^{2}\right), \quad X^{i} \equiv \frac{1}{N R_{(3)}} \sum_{I=1}^{N} x_{(0) I}^{i}, \quad i=1,2, \\
\vec{\rho}=\left(x^{1}, x^{2}\right), \quad \rho^{2}=\sum_{i=1}^{2}\left(x^{i}\right)^{2} .
\end{gathered}
$$

Here $\widetilde{C}$ is a finite constant, $\sigma_{i}$ are Pauli matrices, and $f_{i}(\vec{\rho})$ are some functions of $\vec{\rho}$. Although we do not know the explicit form of $f_{i}(\vec{\rho})$, we know that they fall off exponentially for $\rho>>\widetilde{C}^{-1}$, i.e. outside the core of the soliton. The moduli space of soliton solutions corresponds to the space of solutions for fixed $N$ and $\widetilde{C}$, i.e. fixed asymptotic value of the Higgs (and the other) field(s). It is easily seen from eq.(C.2) that the overall translation in the $x^{1}-x^{2}$ plane corresponds to a shift of $\vec{R}$. Since a change in $\vec{R}$ gives $\delta \phi \propto \delta \vec{R} \cdot \vec{\rho} / \rho^{2}$ asymptotically, the zero modes associated with these deformations are clearly non-normalizable. This shows that the zero modes associated with the $R^{2}$ factor in eq.(4.3) are not normalizable. But this analysis also shows that all other deformations of the solution which keep $\vec{R}$ (and also $\widetilde{C}$ ) fixed give rise to square integrable $\delta \phi$.

Thus in order to test the normalizability of the zero modes corresponding to these other deformations we need to study whether the deformations of the gauge fields induced by these deformations are also square integrable. In this case the gauge fields in fact are not independent, but are related to $\phi$ (up to a gauge transformation) by the Bogomol'nyi equation (A.2). Using this equation, one can verify

$\star$ Note that the center of mass coordinates $X^{1}, X^{2}, X^{3}$ have been scaled by $R_{(3)}$ so that $X^{3}$ has periodicity $2 \pi$. This notation is consistent with the one used in appendix B. 
that the deformations that keep $\widetilde{C}, N$ and $\vec{R}$ in eq.(C.2) fixed induces a gauge field deformations for which $\delta F_{i j} \sim 1 / \rho^{3}$, and hence $\delta A_{i} \sim 1 / \rho^{2}$ asymptotically. Such deformations are clearly square integrable. However we also have deformations of the solution induced by the gauge transformations with parameter $\Lambda$ given in eq.(C.1). This induces an asymptotic gauge field deformation of the form:

$$
\delta A_{i} \propto \frac{N}{2 \pi R_{(3)}} \delta \widehat{\Theta} \sigma_{3} \frac{x^{i}}{\rho^{2}}
$$

This deformation is clearly not square integrable.

Thus we see that the deformations associated with the parameters $X^{1}, X^{2}$ and $\widehat{\Theta}$ are not square integrable, but all other deformations are square integrable. In particular, all deformations associated with the coordinates labelling $\mathcal{M}_{N}^{0}$ are square integrable. Thus the metric on $\mathcal{M}_{N}^{0}$ is finite and hence is independent of the infrared cut-off $L$. On the other hand the metric components $g_{X^{1} X^{1}}, g_{X^{2} X^{2}}$ and $g_{\widehat{\Theta} \widehat{\Theta}}$ are all of order $\ln L$. Using the relation $\Theta=N \widehat{\Theta} \ln L / \pi R_{(3)}$, we see that $g_{\Theta \Theta}$ is of order $(\ln L)^{-1}$.

Even though the metric on $\mathcal{M}_{N}^{0}$ is finite, unlike in the case of four dimensional theory, $\mathcal{M}_{N}^{0}$ does not become flat in the limit when the solitons are far from each other. This is related to the non-normalizability of the zero modes describing the dynamics of single isolated solitons. We shall now derive the form of the metric on the two soliton moduli space in the limit where the two solitons are widely separated. Let $X^{i}(1 \leq i \leq 3)$ denote the center of mass coordinates labelling the space $R^{2} \times S^{1}, \Theta$ be the overall $\mathrm{U}(1)$ phase labelling the second $S^{1}$, and $\widetilde{x}^{i}$, $\tilde{\theta}$ be the relative coordinates labelling the space $\mathcal{M}_{2}^{0}$. We shall normalize the coordinate $\tilde{\theta}$ such that it has periodicity $2 \pi$. Following the analysis given at the end of appendix A, we can show that a change in the solution due to a change in $\widetilde{x}^{3}$ falls off sufficiently fast asymptotically, hence $\widetilde{g}_{33}$ is expected to be finite asymptotically. On the other hand, the asymptotic value of the Higgs field as a 
function of $\vec{R} \equiv\left(R_{(3)} X^{1}, R_{(3)} X^{2}\right)$ and $\overrightarrow{\widetilde{\rho}} \equiv\left(\widetilde{x}^{1}, \widetilde{x}^{2}\right)$ is given by,

$$
\phi \simeq\left\{\widetilde{C}+\frac{1}{\pi R_{(3)}} \ln |\vec{\rho}-(\vec{R}+\overrightarrow{\tilde{\rho}})|+\frac{1}{\pi R_{(3)}} \ln \mid(\vec{\rho}-(\vec{R}-\overrightarrow{\tilde{\rho}}) \mid\} \frac{\sigma_{3}}{2}\right.
$$

If $\delta \phi$ denotes the change of $\phi$ under a variation $\delta \overrightarrow{\widetilde{\rho}}$ of $\overrightarrow{\widetilde{\rho}}$ for fixed $\vec{R}$, then it can easily be verified that,

$$
\int d^{2} \rho|\delta \phi|^{2} \propto \ln \widetilde{\rho}|\delta \overrightarrow{\widetilde{\rho}}|^{2}
$$

with the major contribution to the integral coming from two regions: $|\vec{\rho}-\vec{R}-\overrightarrow{\widetilde{\rho}}|<<$ $\widetilde{\rho}$, and $|\vec{\rho}-\vec{R}+\overrightarrow{\widetilde{\rho}}|<<\widetilde{\rho}$. A similar contribution is obtained from the computation of $\int d^{2} \rho\left|\delta A_{i}\right|^{2}$. This gives $\widetilde{g}_{i j} \propto \ln \widetilde{\rho} \delta_{i j}$ for $1 \leq i, j \leq 2$.

Finally we need to analyze $\widetilde{g}_{\theta \theta}$. In this case we proceed in a somewhat indirect manner. Let $\widetilde{p}_{\theta}$ be the momentum conjugate to $\widetilde{\theta} . \widetilde{p}_{\theta}$ is quantized in integer units since $\tilde{\theta}$ has been normalized to have periodicity $2 \pi$. For large separation between the two solitons, $\widetilde{p}_{\theta}$ has the interpretation as the difference in the electric charges carried by the two solitons. Now, as has been argued at the end of Sec.4, if $\widetilde{p}_{\theta} \neq 0$, i.e. if the two solitons carry different amount of electric charges, then the attractive force between them proportional to $1 / \widetilde{\rho}$ due to the higgs field wins over the repulsive force due to the electromagnetic fields. In the weak coupling limit, this net force is proportional to $\left(\widetilde{p}_{\theta}\right)^{2} / \widetilde{\rho}$. The net result is a contribution to the potential energy (and hence to the Hamiltonian) proportional to $\left(\widetilde{p}_{\theta}\right)^{2} \ln \widetilde{\rho}$. This, in turn, means that the Lagrangian of the system must contain a term proportional to $(\ln \widetilde{\rho})^{-1}(d \widetilde{\theta} / d t)^{2}$. Thus $\widetilde{g}_{\theta \theta} \propto(\ln \widetilde{\rho})^{-1}$.

Combining the above results, we get the following asymptotic form of different components of the metric

$$
\widetilde{g}_{i j} \simeq C_{1} \ln \widetilde{\rho} \delta_{i j}, \quad \widetilde{g}_{33} \simeq C_{2}, \quad \widetilde{g}_{\theta \theta} \simeq C_{3}(\ln \widetilde{\rho})^{-1}
$$

where $C_{i}$ are constants. 
Acknowledgements: I wish to thank J. Cohn, A. Dabholkar, J. Harvey, J. Schwarz, A. Shapere, S. Shenker, C. Vafa and E. Witten for useful discussions. I would also like to acknowledge the hospitality of the Department of Physics at Rutgers university, where part of this work was done.

\section{REFERENCES}

1. C. Montonen and D. Olive, Phys. Lett. B72 (1977) 117; P. Goddard, J. Nyuts and D. Olive, Nucl. Phys. B125 (1977) 1.

2. H. Osborn, Phys. Lett. B83 (1979) 321.

3. A. Font, L. Ibanez, D. Lust and F. Quevedo, Phys. Lett. B249 (1990) 35; S.J. Rey, Phys. Rev. D43 (1991) 526.

4. A. Sen, Nucl. Phys. B404 (1993) 109 (hep-th/9207053), Phys. Lett. B303 (1993) 22 (hep-th/9209016), Mod. Phys. Lett. A8 (1993) 2023 (hepth/9303057); J.Schwarz and A. Sen, Phys. Lett. B312 (1993) 105 (hepth/9305185).

5. J. Schwarz and A. Sen, Nucl. Phys. B411 (1994) 35 (hep-th/9304154).

6. A. Sen, preprint TIFR-TH-94-03 (hep-th/9402002) (to appear in Int. J. Mod. Phys. A), and references therein.

7. A. Sen, Phys. Lett. B329 (1994) 217 (hep-th/9402032).

8. J. Gauntlett and J. Harvey, preprint EFI-94-36 (hep-th/9407111).

9. C. Vafa and E. Witten, preprint HUTP-94-A017 (hep-th/9408074).

10. G. Segal, to appear.

11. J. Horne and G. Moore, preprint YCTP-P2-94 (hep-th/9403058).

12. L. Girardello, A. Giveon, M. Porrati and A. Zaffaroni, preprint NYU-TH94/06/02 (hep-th/9406128).

13. E. Witten and D. Olive, Phys. Lett. B78 (1978) 97. 
14. E. Martinec, Phys. Lett. B171 (1986) 189.

15. A. Dabholkar, G. Gibbons, J. Harvey and F.R. Ruiz, Nucl. Phys. B340 (1990) 33; A. Dabholkar and J. Harvey, Phys. Rev. Lett. 63 (1989) 719.

16. N. Seiberg and E. Witten, preprints RU-94-52 (hep-th/9407087); RU-94-60 (to appear).

17. P. Aspinwall and D. Morrison, preprint DUK-TH-94-68 (hep-th 9404151) and references therein.

18. A. Ceresole, R. D'Auria and S. Ferrara, preprint CERN-TH.7384/94 hepth/9408036).

19. N. Marcus and J. Schwarz, Nucl. Phys. B228 (1983) 145.

20. M. Duff and J. Lu, Nucl. Phys. B347 (1990) 394.

21. A. Giveon, E. Rabinovici and G. Veneziano, Nucl. Phys. B322 (1989) 167; A. Shapere and F. Wilzcek, Nucl. Phys. B322 (1989) 669; M. Rocek and E. Verlinde, Nucl. Phys. B373 (1992) 630 (hep-th/9110053); A. Giveon and M. Rocek, Nucl. Phys. B380 (1992) 128 (hep-th/9112070).

22. P. Candelas, G. Horowitz, A. Strominger and E. Witten, Nucl. Phys. B258 (1985) 46.

23. J. Distler and S. Kachru, Nucl. Phys. B413 (1994) 213 (hep-th/9309110); preprints PUPT-1465 (hep-th/9406090), PUPT-1464 (hep-th/9406091), and references therein.

24. S. Shatashvili and C. Vafa, preprint HUTP-94/A016 (hep-th 9407025).

25. B. Greene, A. Shapere, C. Vafa and S. Yau, Nucl. Phys. B337 (1990) 1.

26. S. Ferrara, C. Kounnas and M. Porrati, Phys. Lett. B181 (1986) 263; M. Terentev, Sov. J. Nucl. Phys. 49 (1989) 713.

27. J. Maharana and J. Schwarz, Nucl. Phys. B390 (1993) 3 (hep-th/9207016); S. Hassan and A. Sen, Nucl. Phys. B375 (1992) 103 (hep-th/9109038). 
28. K. Narain, Phys. Lett. B169 (1986) 41.

29. K. Narain, H. Sarmadi and E. Witten, Nucl. Phys. B279 (1987) 369.

30. J. Gauntlett, J. Harvey and J. Liu, Nucl. Phys. B409 (1993) 363.

31. R. Ward, Phys. Lett. 158B (1985) 424.

32. J. Gauntlett, Nucl. Phys. B400 (1993) 103 (hep-th/9205008); Nucl. Phys. B411 (1994) 443 (hep-th/9305068).

33. E. Witten, Nucl. Phys. B202 (1982) 253.

34. J. Blum, preprint EFI-94-04 (hep-th/9401133).

35. G. Gibbons and P. Ruback, Comm. Math. Phys. 115 (1988) 267; N. Manton and B. Schroers, Annals. of Phys. 225 (1993) 290.

36. K. Muller, Phys. Lett. B177 (1986) 389.

37. M. Duff and R. Khuri, Nucl. Phys. B411 (1994) 473 (hep-th/9305142), Nucl. Phys. B418 (1994) 195 (hep-th/9311120).

38. A. Sen, Int. J. Mod. Phys. A8 (1993) 5079 (hep-th/9302038).

39. M. Atiyah and N. Hitchin, Phys. Lett. 107A (1985) 21; Phil. Trans. R. Soc. Lond. A315 (1985) 459; The Geometry and Dynamics of Magnetic Monopoles, Princeton University Press (1988), and references therein.

40. N. Manton and M. Murray, preprint DAMTP 94-57 (hep-th/9407102). 